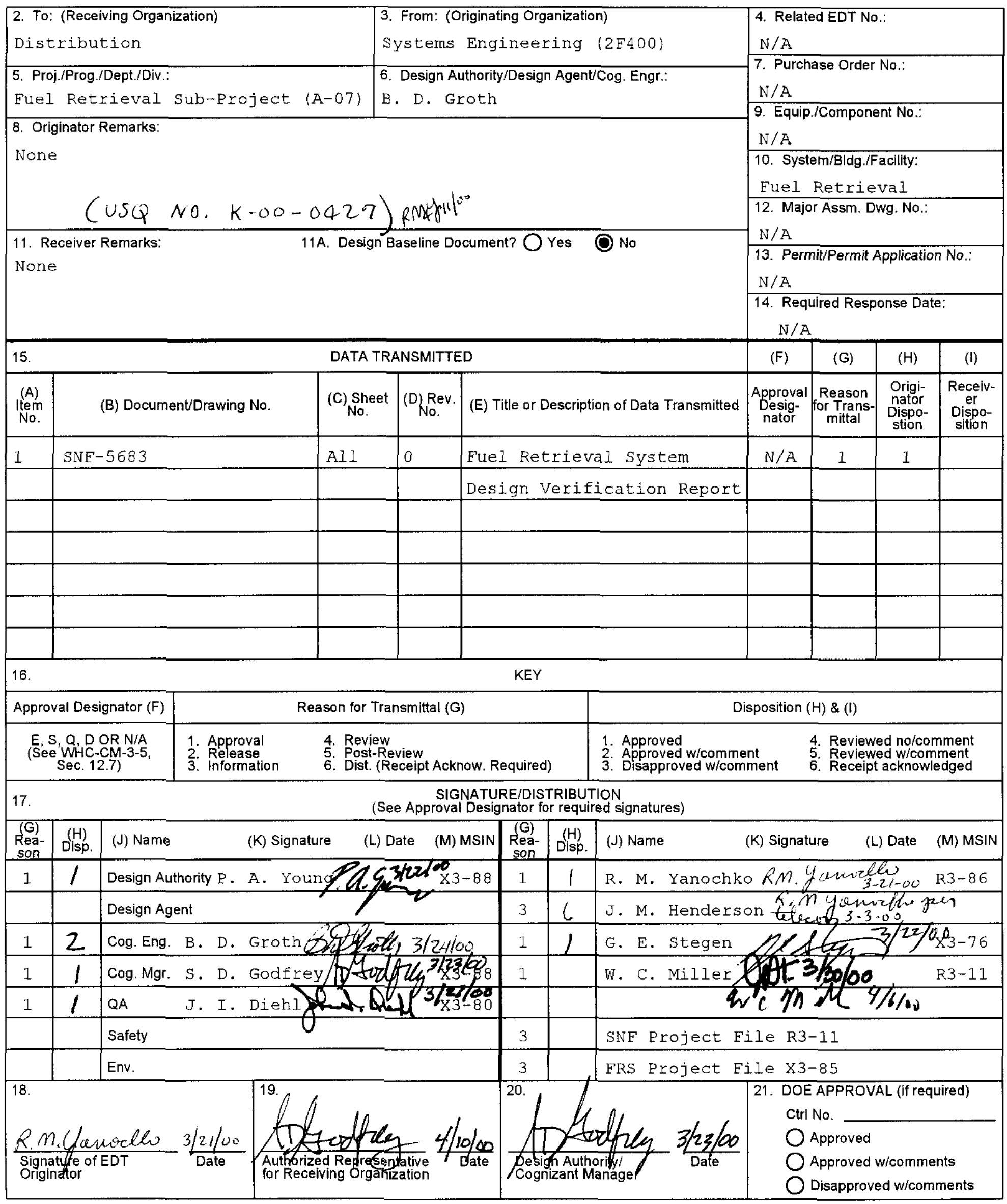


SNF-5683

Revision 0

\section{Fuel Retrieval System Design Verification Report}

Prepared for the U.S. Department of Energy

Assistant Secretary for Environmental Management

Project Hanford Management Contractor for the

U.S. Department of Energy under Contract DE-AC06-96RL13200

Filuor Hanford

P.O. Box 1000

Richland, Washington 
SNF-5683

Revision 0

EDT 623763

\section{Fuel Retrieval System Design Verification Report}

B. D. Groth

ARES Corporation

R. M. Yanochko

Fluor Hanford

Date Published

March 2000

Prepared for the U.S. Department of Energy

Assistant Secretary for Environmental Management

Project Hanford Management Contractor for the

U.S. Department of Energy under Contract DE-AC06-96RL13200

Fluor Hanford

P.O. Box 1000

Richland, Washington
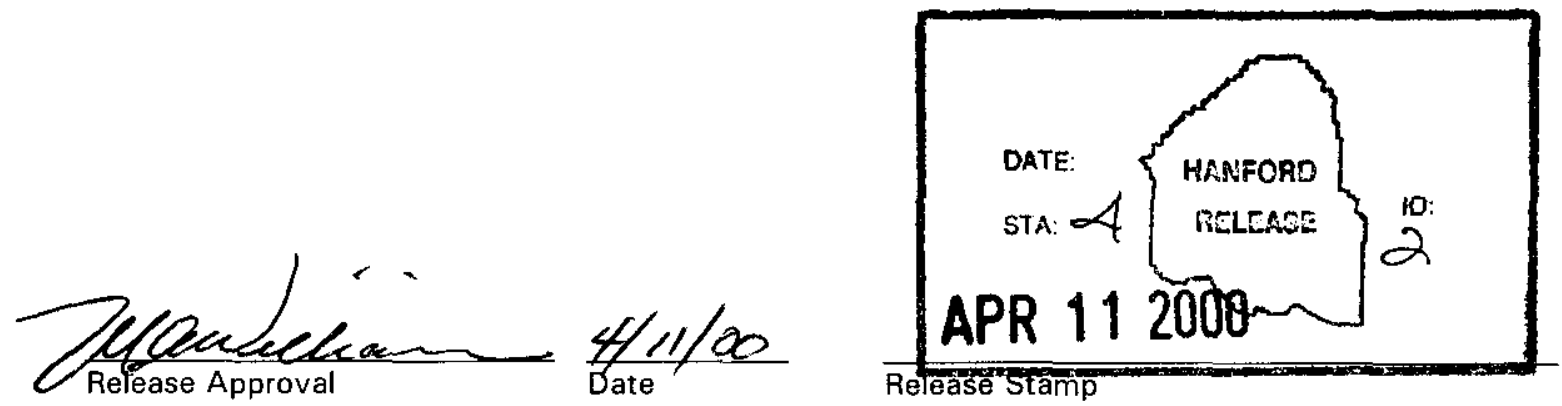
TRADEMARK DISCLAIMER

Reference herein to any specific commercial product, process, or service by trade name, trademark, manufacturer, or otherwise, does not necessarily constitute or imply its endorsement, recommendation, or favoring by the United States Government or any agency thereof or its contractors or subcontractors.

This report has been reproduced from the best available copy. Available in paper copy and microfiche.

Available electronically at http://www.doe.gov/bridge. Available for a processing fee to the U.S. Department of Energy and its contractors, in paper, from:

U.S. Department of Energy

Office of Scientific and Technical Information

P.O. Box 62

Oak Ridge, TN 37831-0062

phone: $865-576-8401$

fax: 865-576-5728

email: reports@adonis.osti.gov(423) 576-8401

Printed in the United States of America

Total Pages: $\quad 2 / 3$

SNF. S683, RWo 


\section{SNF-5683, Revision 0}

\section{Table of Contents}

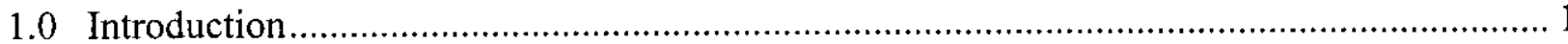

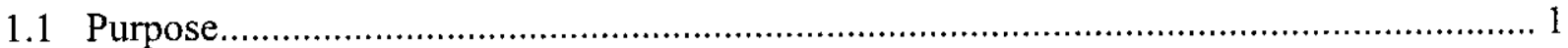

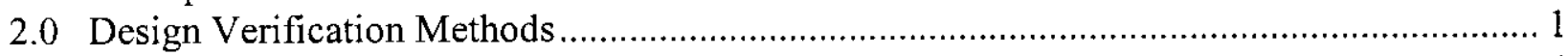

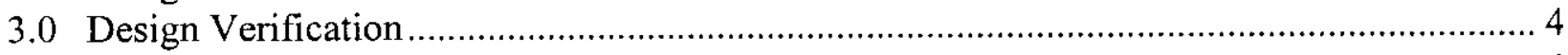

3.1 Design Verification Compliance Results.................................................................. 4

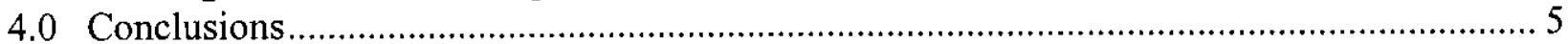

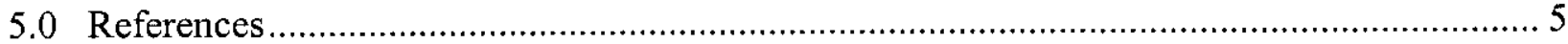

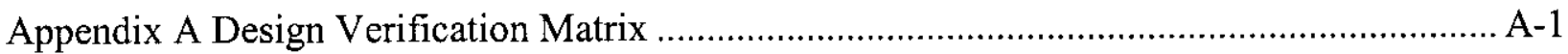

Appendix B FRS Technical Document Status.................................................................... B-1

Appendix C Fuel Retrieval System ALARA and Radiological Design Basis and Review

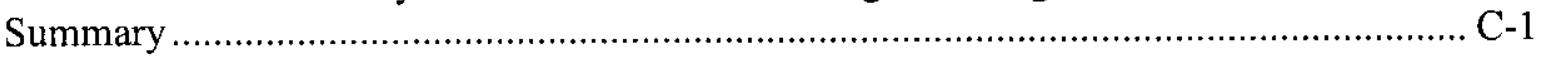

\section{List of Tables}

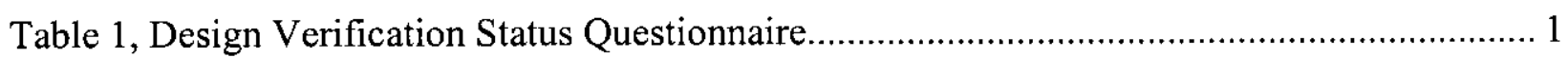

Table A-1 Design Verification Matrix ........................................................................... A-2

Table B-1 FRS Technical Document Status ........................................................................ B-2

\section{List of Acronyms}

This section defines acronyms used in the document text.

BNFL British Nuclear Fuels Ltd.

CVI Certified Vendor Information

DT/R Data Transmittal and Review form

ECN Engineering Change Notice

EDT Engineering Data Transmittal

FRS Fuel Retrieval System

ORR operational readiness review

SEL Safety Equipment List

SNF Project Spent Nuclear Fuel Project

SSC structures, systems, and components

TDC Technical Document Control 


\subsection{Introduction}

The Fuel Retrieval Subproject was established as part of the Spent Nuclear Fuel Project (SNF Project) to retrieve and repackage the SNF located in the K Basins. The Fuel Retrieval System (FRS) construction work is complete in the $\mathrm{KW}$ Basin, and start-up testing is underway. Design modifications and construction planning are also underway for the KE Basin. An independent review of the design verification process as applied to the $\mathrm{K}$ Basin projects was initiated in support of preparation for the SNF Project operational readiness review (ORR).

A Design Verification Status Questionnaire, Table 1, is included which addresses Corrective Action SNF-EG-MA-EG-20000060, Item \#9 (Miller 2000).

\section{$1.1 \quad$ Purpose}

This report provides evidence of completed verification activities demonstrating FRS requirements have been met as described in SNF-5471, Fuel Retrieval System Design Verification (Groth, Henderson, Yanochko, 2000).

This report identifies design verification completion references, FRS design baseline documents, and incomplete verification activities.

\subsection{Design Verification Methods}

FRS verification methods are based upon SSC Quality Levels designated and described in SNF5471 (Groth, Henderson, Yanochko, 2000).

Safety classification of Systems, Structures and Components (SSCs) are documented in the SNF Fuel Retrieval Subproject Safety Analysis Document (Bergmann, 1998). FRS safety classifications will need to be incorporated in to the K Basins Safety Equipment List (SEL). 
SNF-5683, Revision 0

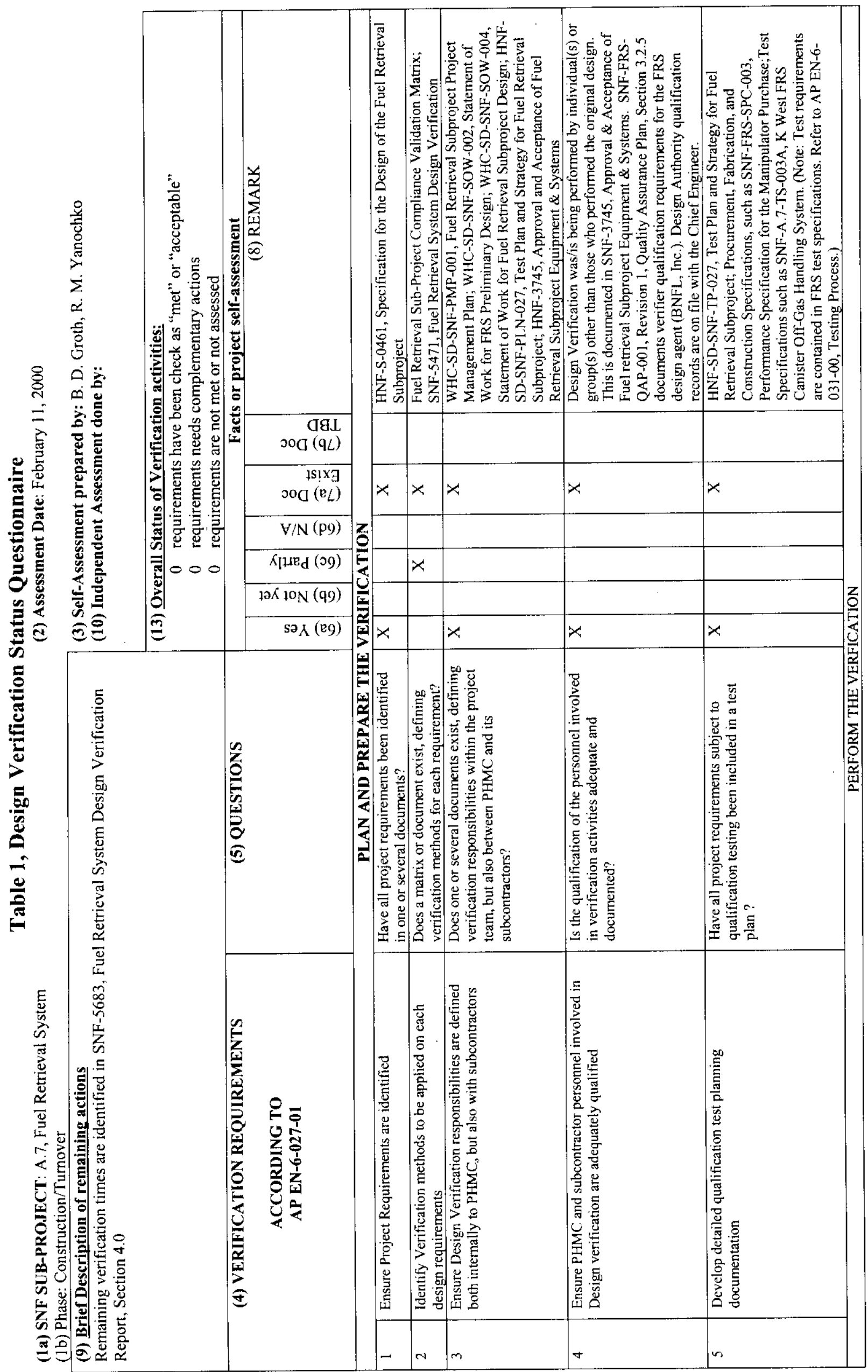


SNF-5683, Revision 0

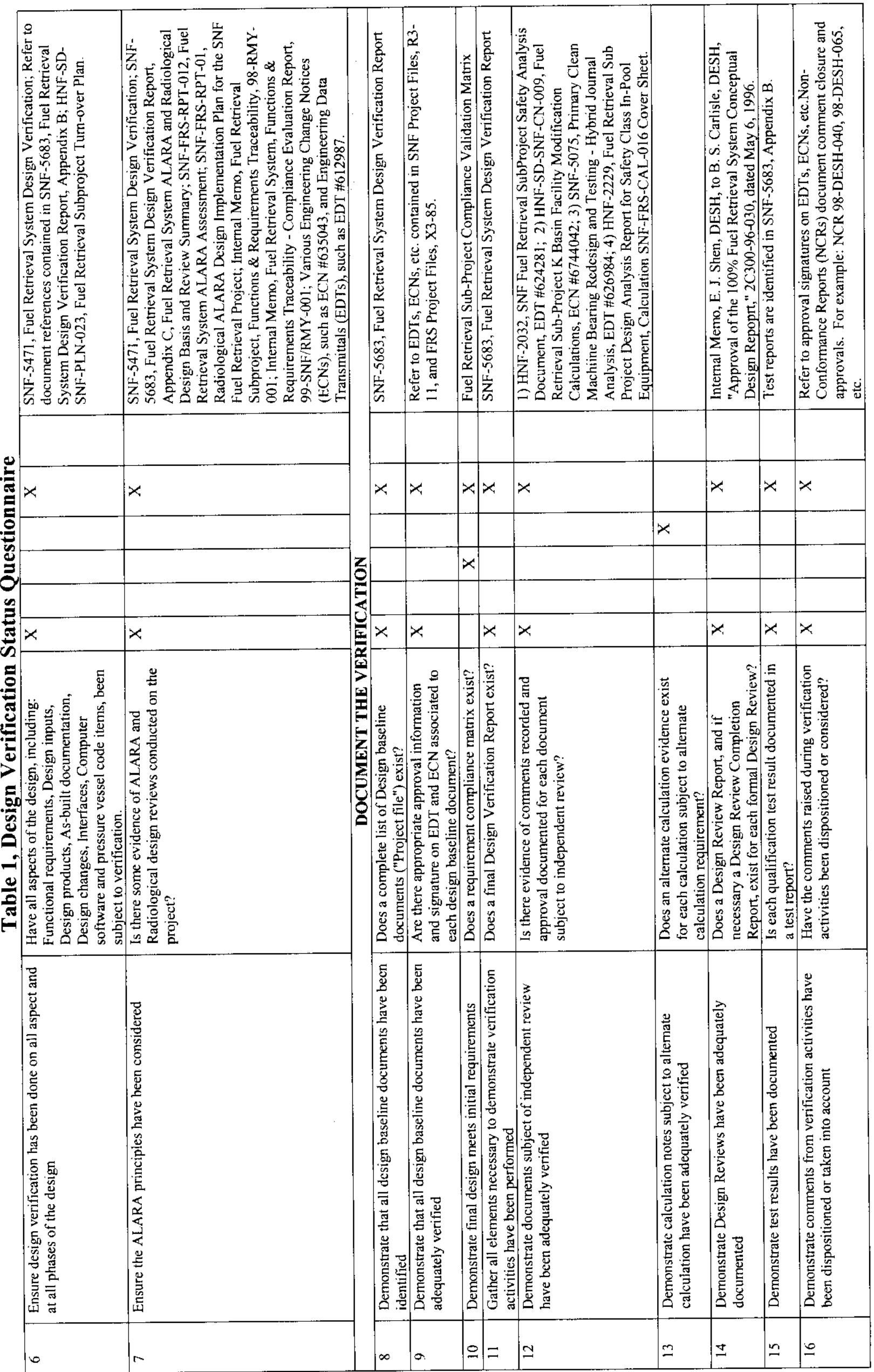




\subsection{Design Verification}

A description of FRS design verification activities is contained in SNF-5471 (Groth, Henderson, Yanochko, 2000). SNF-5471 describes other verification activities not identified herein. A listing of verification completion references is contained in Appendix A, Table A-1, which needs to be worked with the Compliance Evaluation Report as described in SNF-5471, Section 4.1 to determine how individual requirements were verified. FRS design verification activities conducted at the major component level, are identified in SNF-5471, Table C-1, FRS Structures, Systems, and Components Design Verification Method (Groth, Henderson, Yanochko, 2000). The documents that provide evidence of the completed verification are identified in Appendix A, Table A-1, of this report. For the majority of the components, the final design was verified through informal design reviews and factory acceptance testing. In addition, functional performance of active components and systems will be verified in their installed configuration through a series of pre-operational acceptance tests. The fuel cleaning performance of the primary clean machine system will then be validated with actual spent nuclear fuel. The objectives of FRS testing are identified in HNF-SD-SNF-TP-027, Test Plan and Strategy for the Fuel Retrieval Subproject. These objective summary follows:

Process Validation Testing - Verifies full operational capability of the FRS with actual fuel, and to establish the process operational baseline for fuel cleaning and MCO fuel and scrap basket loading.

Factory Acceptance Testing - Provides verification that the equipment conforms to requirements called out in the procurement specifications for fit, form, and function.

Construction Acceptance Testing - Provides verification that the equipment is installed in conformance with the construction specification and drawings with focus on fit and function.

Informal reviews and approval of vendor design submittals are documented on individual Data Submittal and Review forms (DT/Rs) by the reviewer's signature. These original DT/Rs are retained by Technical Document Control (TDC). TDC then consolidates any reviewer comments for transmittal to the vendor, indicates document approval if appropriate, and distributes the document as directed.

Signatures on Engineering Data Transmittals (EDTs) and on Engineering Change Notices (ECNs) and drawings are evidence of verification by informal review of Hanford Site documents and drawings.

\subsection{Design Verification Compliance Results}

Evidence of design verification completion is identified in Appendix A, Table A-1, for design verification methods identified in SNF-5471, FRS Design Verification, Table C-1 (Groth, Henderson, Yanochko, 2000). Fuel Retrieval System ALARA and Radiological Design Basis is described in Appendix C. 
FRS technical documents are listed in Appendix B along with their status. The document list is organized using File Index Numbers corresponding to the FRS Project Working File system. Information not technically related is not included. Therefore, breaks in the File Index numbering will occur. Categories of technical information included in Appendix $\mathrm{C}$ is shown below. The File Index identifier is followed by the category of documentation.

1.1 Project Management Plans

1.2 Technical Baseline documentation such as, specifications, plans, design documents, analyses, and reports.

1.4 FRS Procurement, Fabrication, \& Construction documentation such as vendor information.

1.5 FRS Test and Turnover documentation such as, test plans, test specifications, procedures, etc.

1.6 Regulatory/Safety Activity documentation such as, plans, radiological and safety analyses, reports, etc.

\subsection{Conclusions}

If verification completion action is required, the entry in the "Notes" column of Appendix B Table is bolded. Open items that require action include:

- HNF-3637, which is the development control workplan for the stiff-back and empty basket grapples, needs to be re-released with the required DA and QA signatures.

- Final as-built drawings and documents need to be entered in the CVI files. Fabrication and installation may be complete for a system, but final as-builts are still being prepared. This will be closed as part of the ABU checklist completion.

- Design activities are still being conducted on the In-Pool Equipment by Westinghouse Engineered Products Division (DAG series drawings). Recent drawings will need to be entered in the Technical Document List.

- In additional, functional performance of active components and systems will be verified in their installed configuration through a series of preoperational acceptance tests. The fuel cleaning performance of the primary clean machine system will then be validated with actual spent nuclear fuel.

\subsection{References}

Bergman, T.B., 1999, Management Assessment Report of Subproject Design Verification Activities, memo 99-SNF/TBB-001, Fluor Hanford, Inc., Richland, Washington.

Bergmann, D.W., 1998, SNF Fuel Retrieval Subproject Safety Analysis Document, HNF-2032, Rev. 0, Duke Engineering and Services Hanford, Inc., Richland, Washington. 
SNF-5683, Revision 0

FDH, 1999, Design Verification Process, AP EN-6-027, Rev. 1, Fluor Daniel Hanford, Inc., Richland, Washington.

Groth, Henderson, Yanochko, 2000, Fuel Retrieval System Design Verification, SNF-5471, Rev. 0, Fluor Hanford, Inc., Richland, Washington.

Miller, W. C., 2000, Corrective Action SNF-EG-MA-EG-20000060, Item \#9, 00-SNF/WCM-011, February 7, 2000. 
SNF-5683, Revision 0

Appendix A

Design Verification Matrix

A-1 


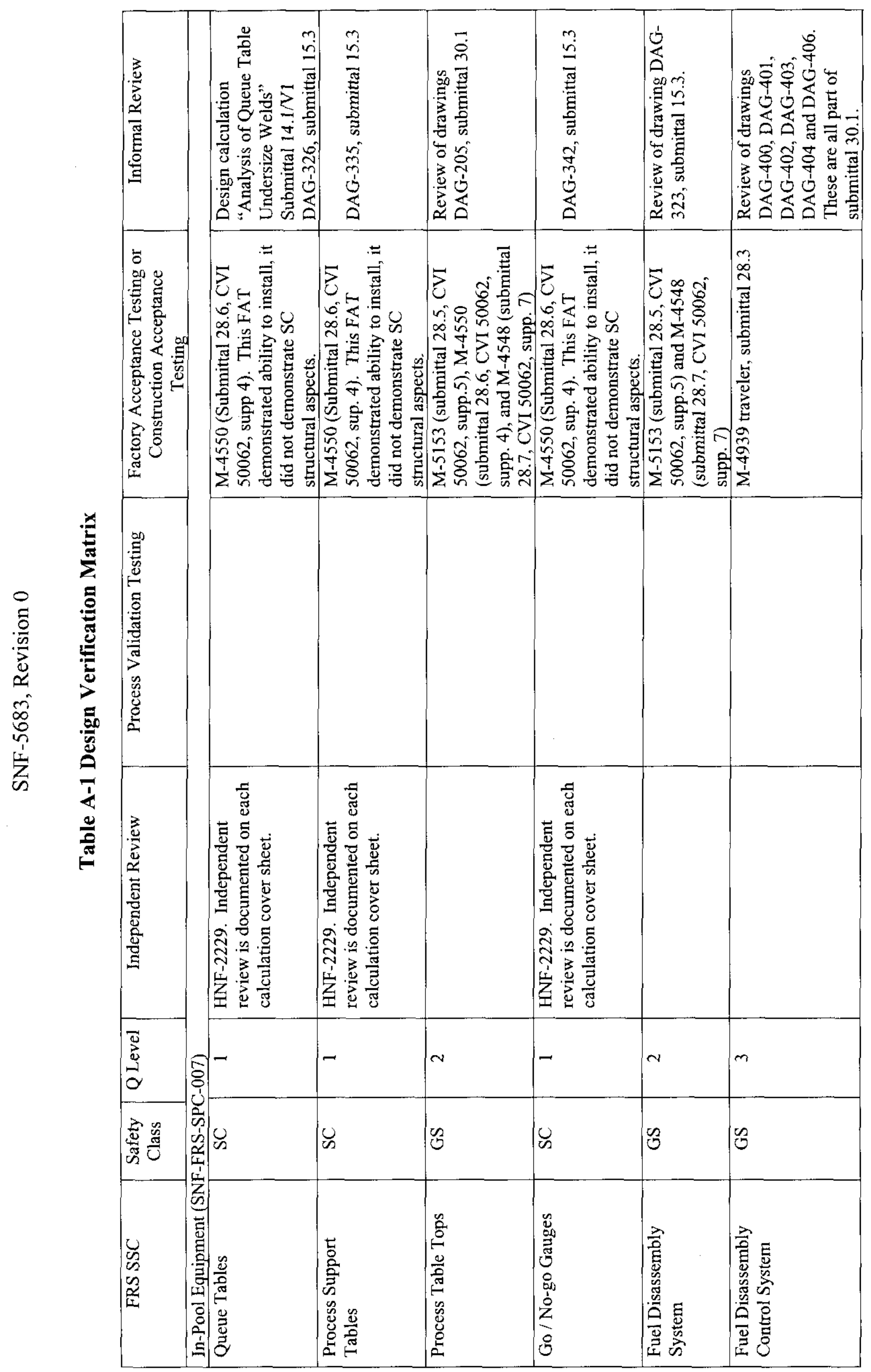




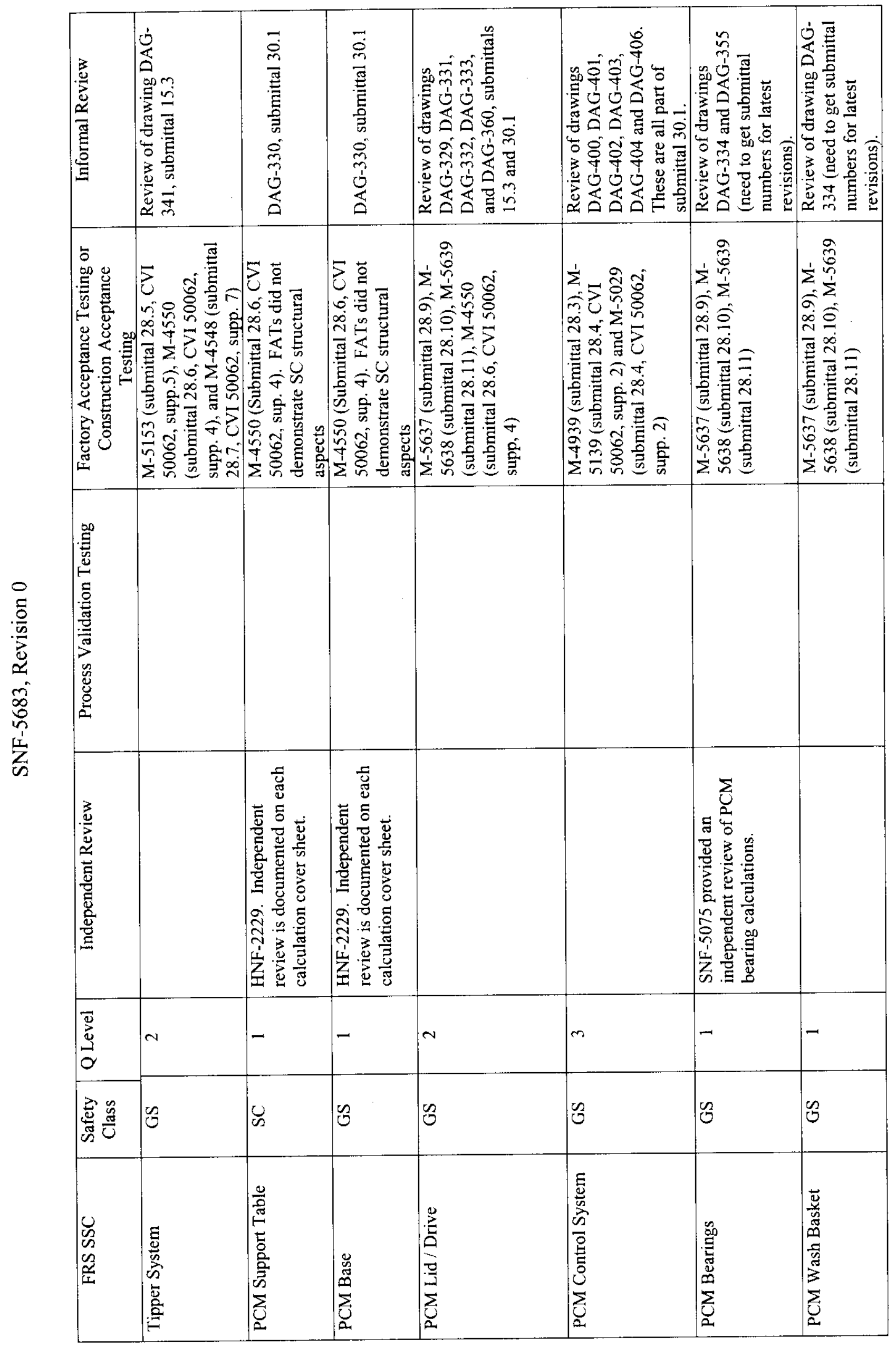




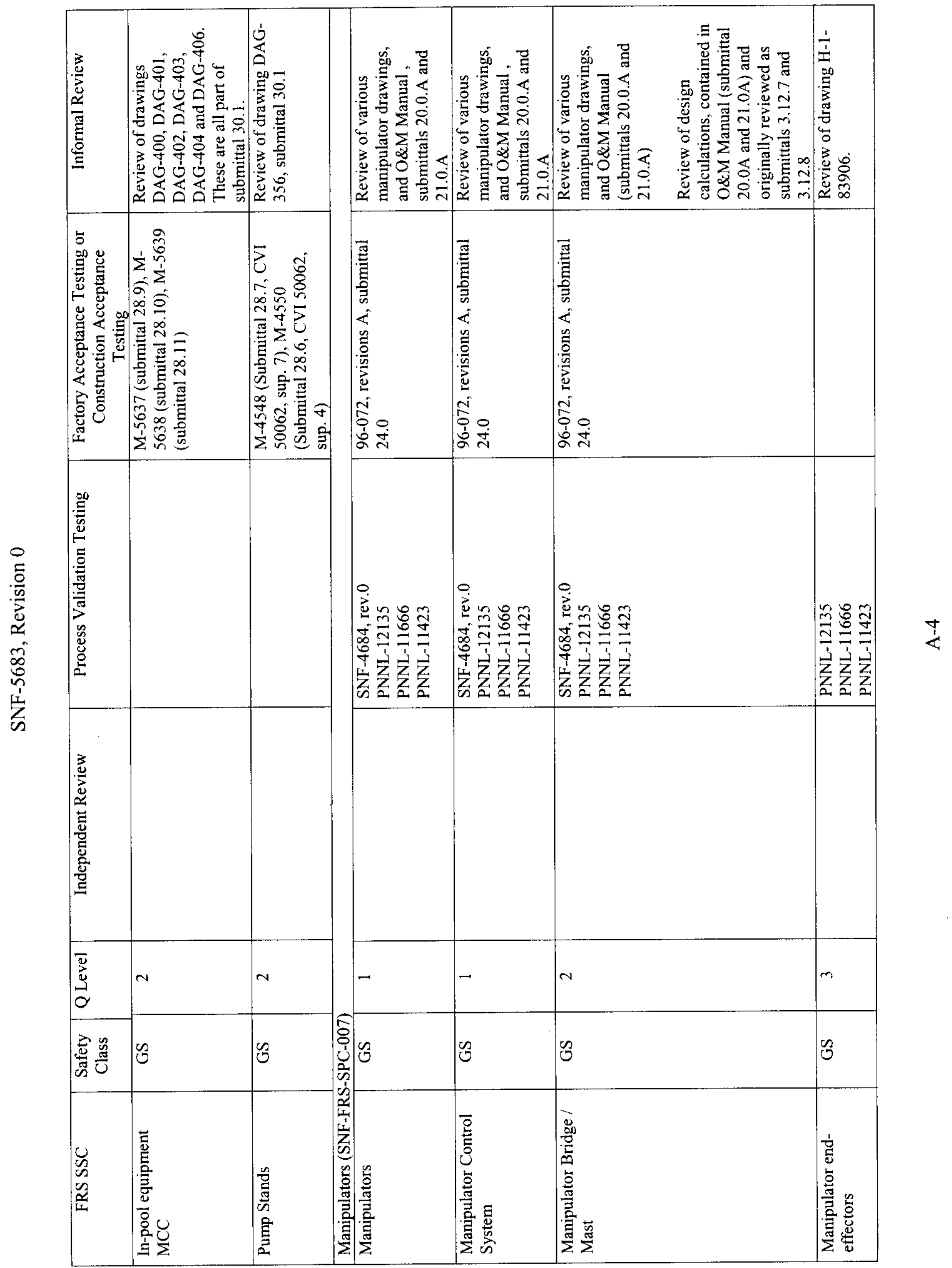




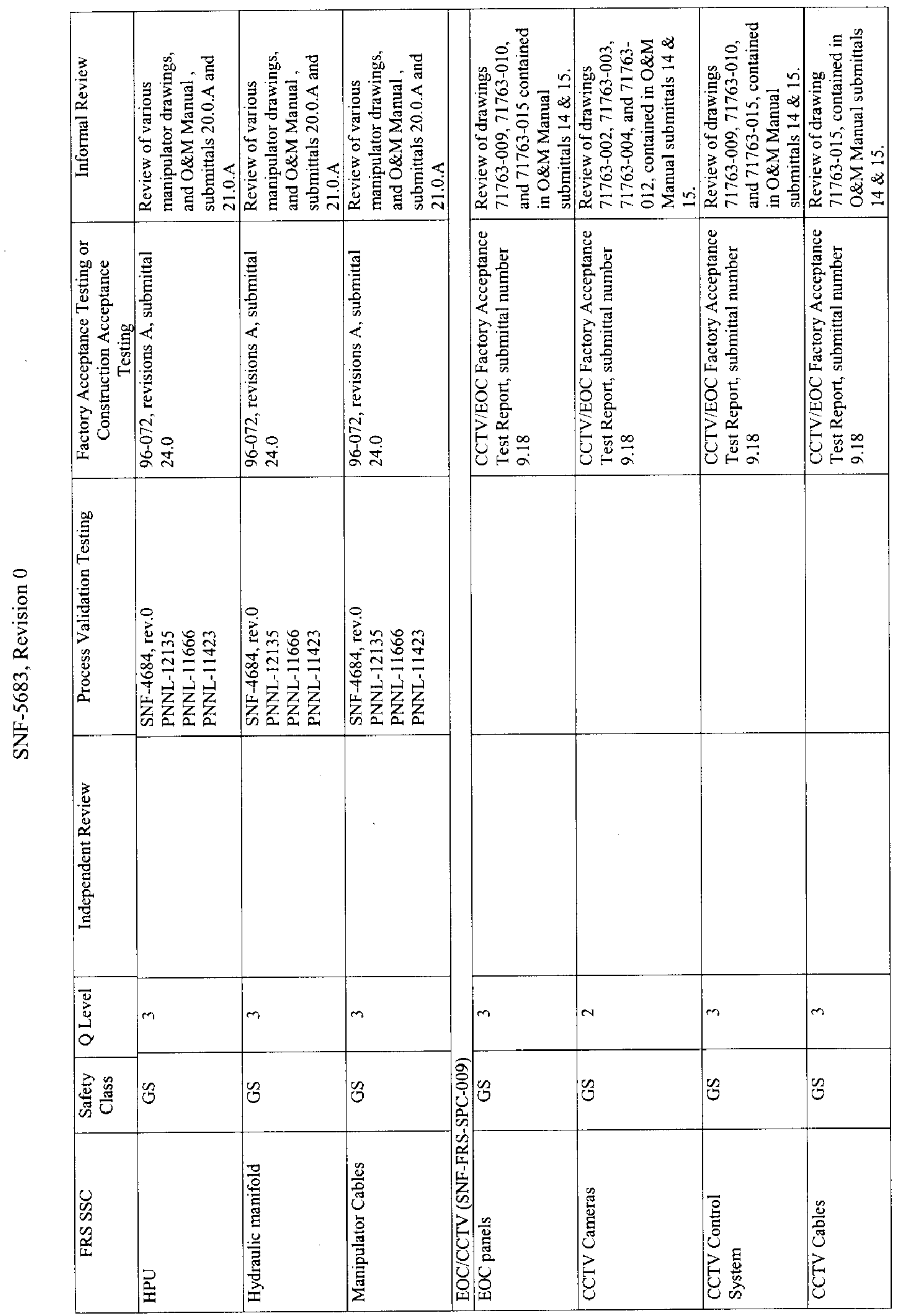




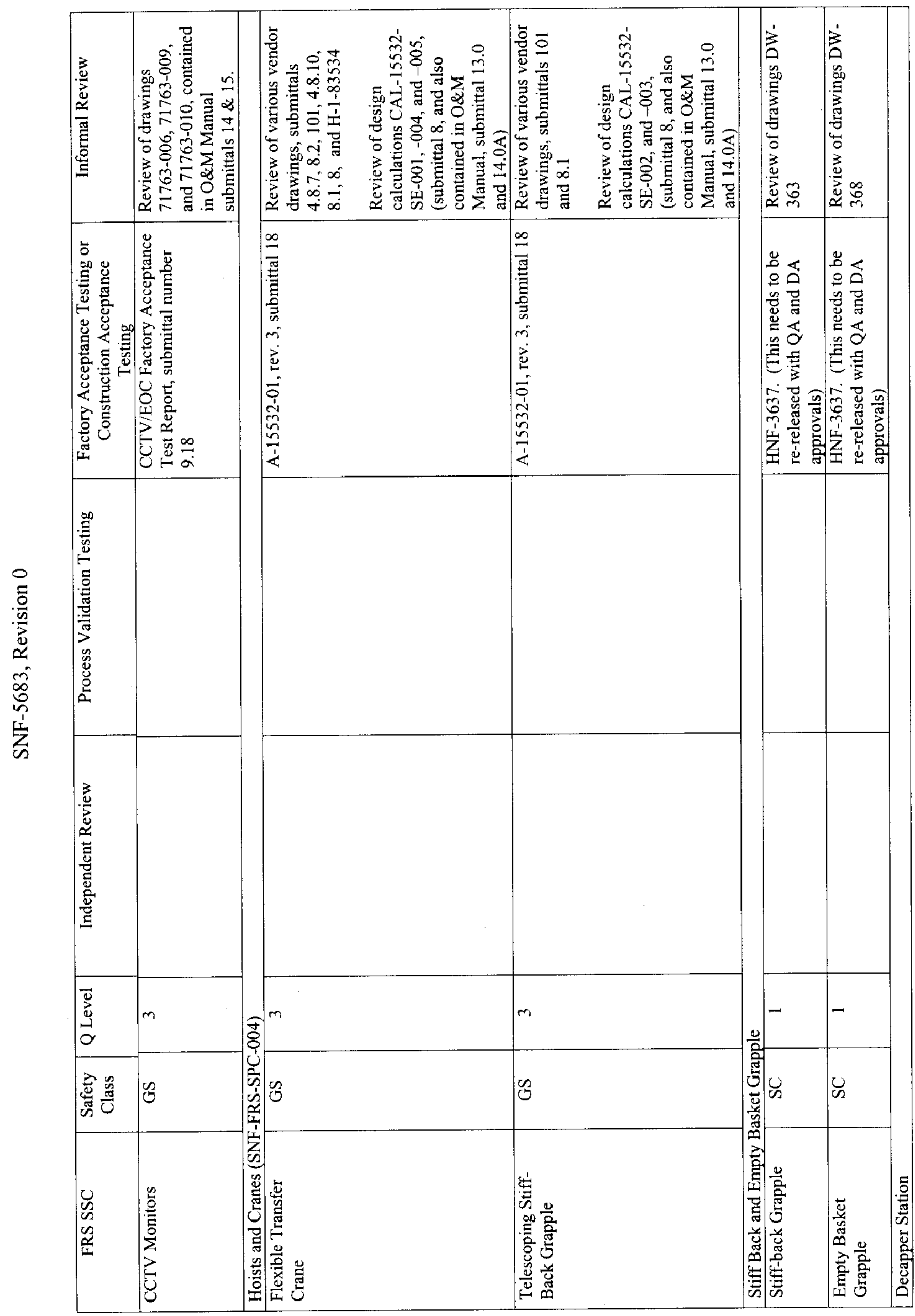

1
4 


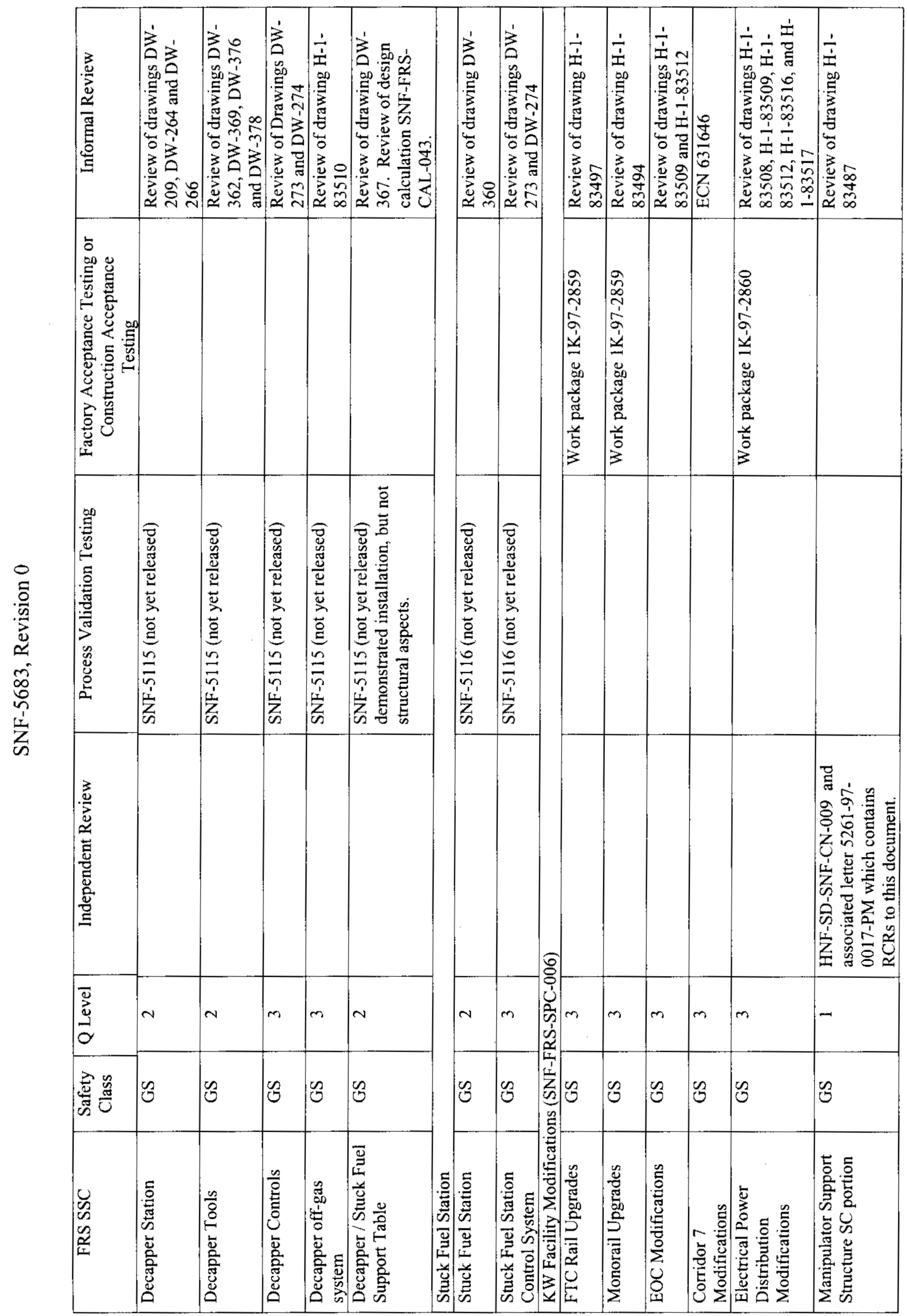




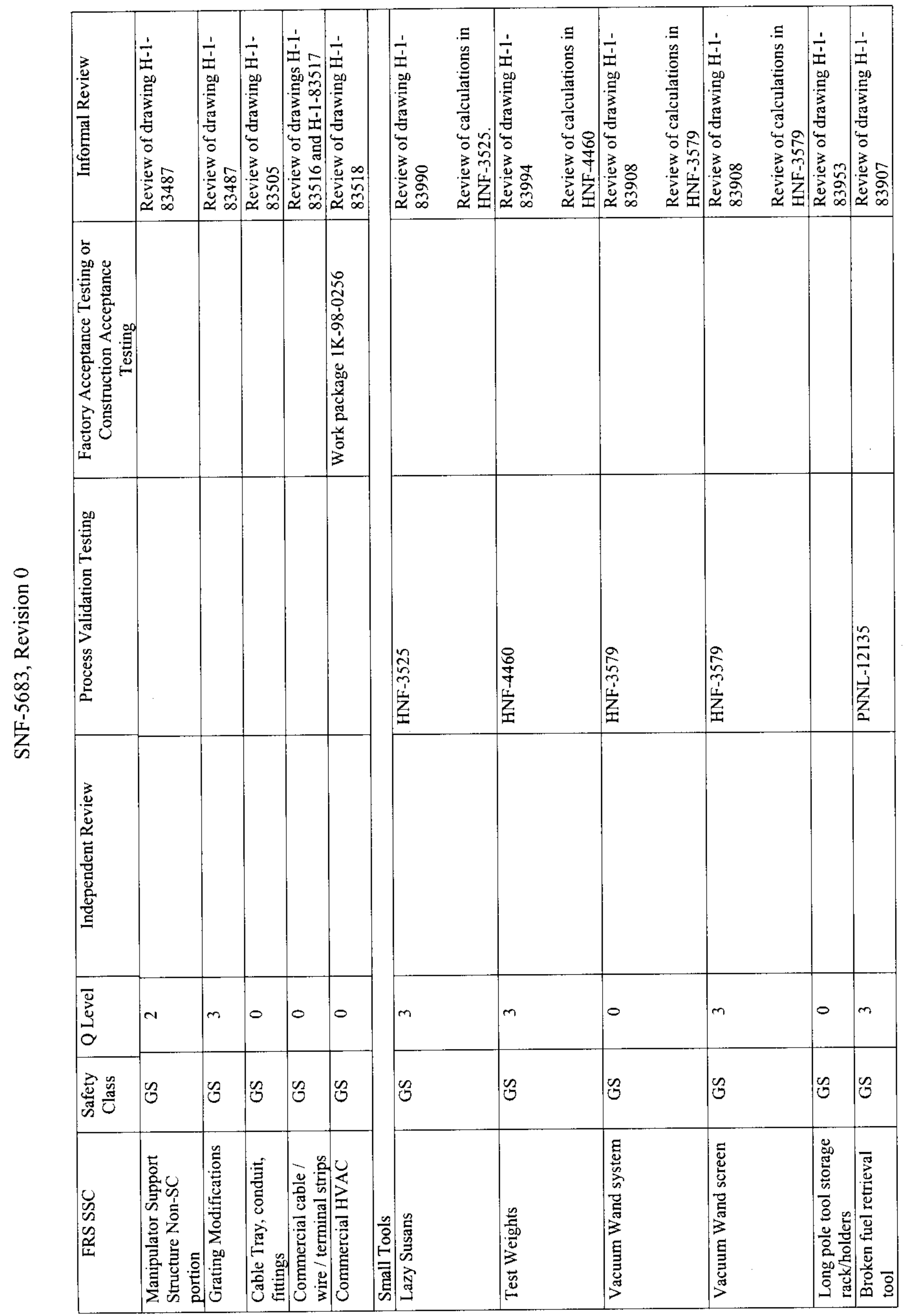




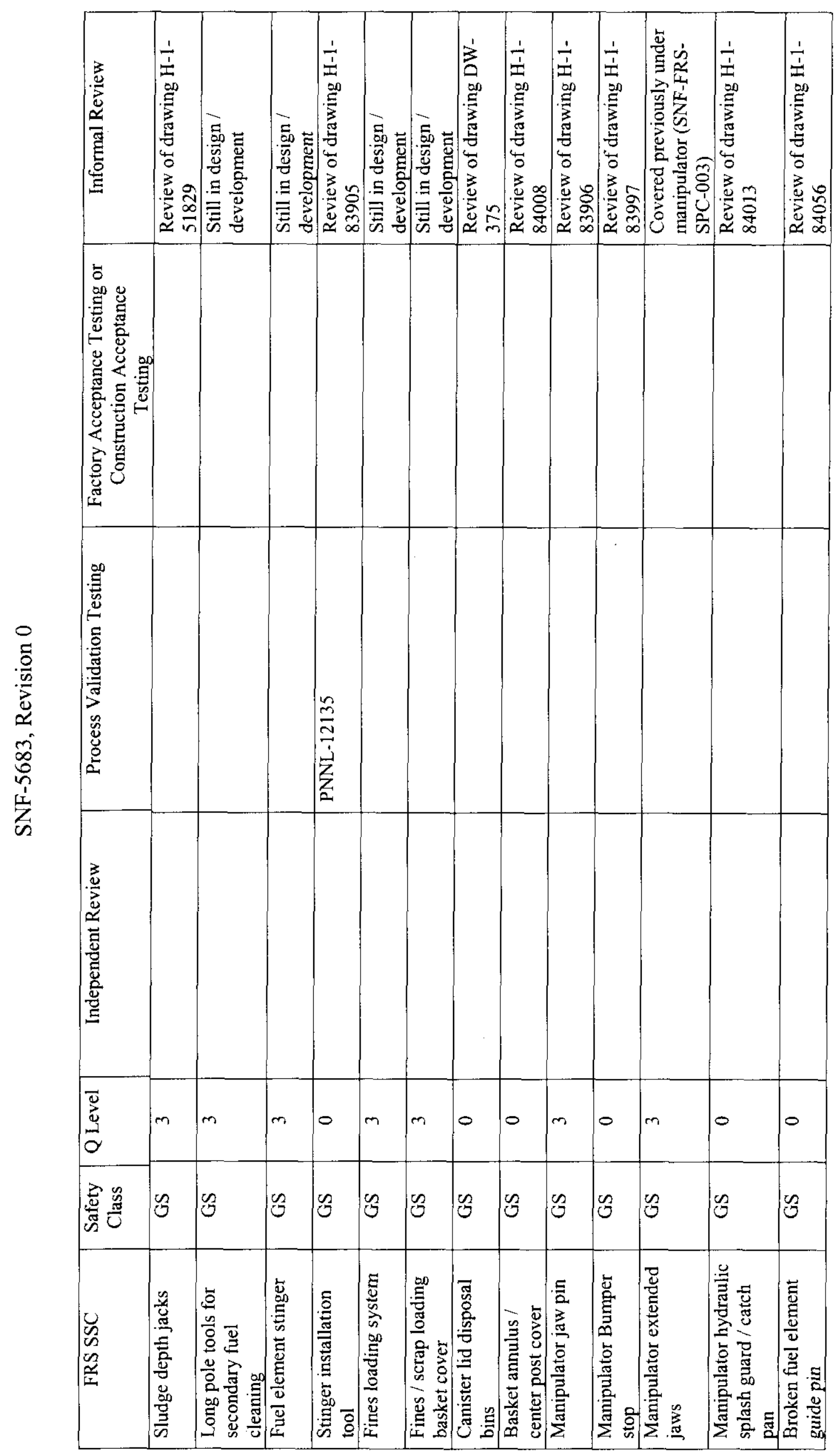




\section{References:}

HNF-2229, Rev. 0, Fuel Retrieval Subproject Design Analysis Report for Safety Class In-Pool Equipment, Duke Engineering and Services Hanford, Richland, Washington.

M-4550, Rev. 0, Dry Installation/Maintenance Checkout of PCM and Process Support Tables, Table Tops, PCM Base, PCM Lid, and PCM Lid Hinges, Submittal 28.6, CVI 50062, sup. 4, Westinghouse Engineered Products Division, Carlsbad, New Mexico.

Analysis of Queue Table Undersize Welds, Submittal 14.1/V1, Westinghouse Engineered Products Division, Carlsbad, New Mexico.

M-5153, Rev. 0, Dry Factory Acceptance Testing of the Process Table Equipment, Table 6-3, Test No. 6.3.1.1 - 6.3.1.7, Submittal 28.5, CVI 50062, sup. 5, Westinghouse Engineered Products Division, Carlsbad, New Mexico.

M-4548, Rev. 0, Installation / Maintenance Testing of the Process Equipment, Submittal 28.7, CVI 50062, sup. 7, Westinghouse Engineered Products Division, Carlsbad, New Mexico.

DAG-205, Rev. 8, Process Table Assembly, Submittal 30.1, Westinghouse Engineered Products Division, Carlsbad, New Mexico.

DAG-323, Rev. 5, Disassembler Station, Submittal 15.1, Westinghouse Engineered Products Division, Carlsbad, New Mexico.

M-4939, Rev. 0, In-Pool Equipment Control System Test Report, Submittal 28.3, Westinghouse Engineered Products Division, Carlsbad, New Mexico.

DAG-400, Rev. 3, FRS In-Pool Equipment Control System Cover Sheet, Submittal 30.1, Westinghouse Engineered Products Division, Carlsbad, New Mexico.

DAG-401, Rev. 3, System power Distribution One Line Diagram, Submittal 30.1, Westinghouse Engineered Products Division, Carlsbad, New Mexico.

DAG-402, Rev. 6, FRS In-Pool Equipment Panel Layouts, Submittal 30.1, Westinghouse Engineered Products Division, Carlsbad, New Mexico.

DAG-403, Rev. 5, FRS In-Pool Equipment Control System Electrical Schematic, Submittal 30.1, Westinghouse Engineered Products Division, Carlsbad, New Mexico.

DAG-404, Rev. 3, FRS In-Pool Equipment Control System Interconnect Diagram, Submittal 30.1, Westinghouse Engineered Products Division, Carlsbad, New Mexico.

DAG-406, Rev. 4, FRS In-Pool Equipment Local Control Panel Wiring Harness, Submittal 30.1, Westinghouse Engineered Products Division, Carlsbad, New Mexico.

M-5637, Rev. 0, K-Basin West Pre-and Post - Testing for Hybrid Journal Design, Submittal 28.9, Westinghouse Engineered Products Division, Carlsbad, New Mexico. 
SNF-5683, Revision 0

DAG-341, Rev. 5, Tipping Station Assembly, Submittal 15.1, Westinghouse Engineered Products Division, Carlsbad, New Mexico.

DAG-329, Rev. 3, PCM Lid Assembly, Submittal 15.3, Westinghouse Engineered Products Division, Carlsbad, New Mexico.

DAG-331, Rev. 2, PCM Lid Drive Assembly, Submittal 15.3, Westinghouse Engineered Products Division, Carlsbad, New Mexico.

DAG-332, Rev. 1, PCM Lid Cylinder Assembly, Submittal 30.1, Westinghouse Engineered Products Division, Carlsbad, New Mexico.

DAG-333, Rev. 1, PCM Hinge Assembly, Submittal 30.1, Westinghouse Engineered Products Division, Carlsbad, New Mexico.

DAG-360, Rev. 4, Primary Clean Machine Drive Assembly, Submittal 15.3, Westinghouse Engineered Products Division, Carlsbad, New Mexico.

M-5139, Rev. 0, Software and Control Equipment Verification, Submittal, 28.4, CVI 50062, sup. 2, Westinghouse Engineered Products Division, Carlsbad, New Mexico.

M-5029, Rev. 0, Checkout of Control System Failure Action, Submittal, 28.4, CVI 50062, sup. 2, Westinghouse Engineered Products Division, Carlsbad, New Mexico.

M-5637, Rev. 0, K-Basin West Pre-and Post-Testing for Hybrid Journal Design, Submittal 28.9, Westinghouse Engineered Products Division, Carlsbad, New Mexico.

M-5638, Rev. 0, K-Basin West Test Hybrid Journal Design with PCM Lid Open, Submittal 28.10, Westinghouse Engineered Products Division, Carlsbad, New Mexico.

M-5639, Rev. 0, K-Basin West Test Hybrid Journal Design with PCM Lid Open/Close, Submittal 28.11, Westinghouse Engineered Products Division, Carlsbad, New Mexico.

DAG-334, Rev. 5, PCM Wash Basket Assembly, Submittal 30.1, Westinghouse Engineered Products Division, Carlsbad, New Mexico.

DAG-355, Rev. 3, PCM Bearing Assembly \& Details, Submittal 30.1, Westinghouse Engineered Products Division, Carlsbad, New Mexico.

SNF-4684, Rev. 0, FRS Manipulator System Maintenance and Upgrade Acceptance Test Report, Duke Engineering and Services Hanford, Richland, Washington.

PNNL-12135, Rev. 0, Final Report SNF Retrieval System Manipulator System Cold Validation Testing, Battelle Pacific Northwest National Laboratory, Richland, Washington.

PNNL-1 1666, Rev. 0, Final Report - Spent Nuclear Fuel Retrieval System Fuel Handling Development Testing, Battelle Pacific Northwest National Laboratory, Richland, Washington. 
SNF-5683, Revision 0

PNNL-11423, Rev. 0, Interim Report - Spent Nuclear Fuel Retrieval System Fuel Handling Development Testing, Battelle Pacific Northwest National Laboratory, Richland, Washington.

96-072, Rev. A, Fuel Retrieval Tele-Operated Manipulator and Hydraulic Power Units Factory Acceptance Test Plan - Final Report (MANIP-FRS-105-A and 105-B), Submittal 24.0, Schilling Robotic Systems, Davis, California.

Konan Manipulator Operations and Maintenance Manual, Submittals 20.0.A and 21.0.A, Schilling Robotic Systems, Davis, California.

CCTV/EOC Technical Manual, Submittals 14 and 15, Oceaneering Hanford Company, Richland, Washington.

CCTV/EOC Factory Acceptance Test Report, Submittal 9.18, Oceaneering Hanford Company, Richland, Washington.

A-15532-01, Rev. 3, FTC Factory Acceptance Test Report, Submittal 18, American Crane Company, Douglasville, Pennsylvania

FTC Operations and Maintenance Manual, Submittals 13.0, 14.A, and 15.1, American Crane Company, Douglasville, Pennsylvania

HNF-3637, Rev. 0, Fuel Retrieval Sub-Project Engineering Work Plan for the Stiff Back and Empty Basket Grapple Design Development, Duke Engineering and Services Hanford, Richland, Washington.

DW-363, Rev. 3, Stiffback Basket Grapple Assembly, Submittal 108, British Nuclear Fuels Ltd., Richland Washington.

DW-368, Rev. 3, Empty MCO Basket Grapple Assembly, Submittal 108, British Nuclear Fuels Ltd., Richland Washington.

DW-209, Rev. 4, FRS In-Pool Equipment Decapping Station, Submittal 108, British Nuclear Fuels Ltd., Richland Washington.

DW-264, Rev. 1, Decapping Station Hydraulic Manifold, Submittal 108, British Nuclear Fuels Ltd., Richland Washington.

DW-266, Rev. 1, Decapping Station Pump Suction Hose, Submittal 108, British Nuclear Fuels Ltd., Richland Washington.

DW-362, Rev. 3, Canister Lid Removal Tool, Submittal 108, British Nuclear Fuels Ltd., Richland Washington.

DW-369, Rev. 3, Canister Lid Removal Tool Deployment System, Submittal 108, British Nuclear Fuels Ltd., Richland Washington. 
SNF-5683, Revision 0

DW-367, Rev. 2, Support Table Decapping and Stuck Fuel Station, Submittal 108, British Nuclear Fuels Ltd., Richland Washington.

DW-273, Rev. 1, Stuck Fuel and Decapping Station Hydraulic System K-West, Submittal 108, British Nuclear Fuels Ltd., Richland Washington.

DW-274, Rev. 2, Stuck Fuel Station Control Panel Front View, Submittal 108, British Nuclear Fuels Ltd., Richland Washington.

DW-360, Rev. 1, FRS In-Pool Equipment Stuck Fuel Station, Submittal 109, British Nuclear Fuels Ltd., Richland Washington.

H-1-83510, Rev. 0, Canister Gas Venting, Fluor Daniel Northwest, Richland, Washington.

SNF-5115, Rev. 0, Fuel Retrieval Sub-Project Decapping Station Performance Test Data Report, Fluor Daniel Hanford, Inc., Richland, Washington.

SNF-5116, Rev. 0, Fuel Retrieval Sub-Project Stuck Fuel Station Performance Test Data Report, Fluor Daniel Hanford, Inc., Richland, Washington.

H-1-83497, Rev. 1, FRS - KW Flexible Crane Upgrade, Fluor Daniel Northwest, Richland, Washington.

H-1-83494, Rev. 1, FRS - KW Monorail Upgrade, Fluor Daniel Northwest, Richland, Washington.

H-1-83509, Rev. 2, FRS - KW EOC Lighting, Fluor Daniel Northwest, Richland, Washington.

H-1-83512, Rev. 2, FRS - KW Electrical Power Plan, Fluor Daniel Northwest, Richland, Washington.

H-1-83508, Rev. 2, FRS - KW Cable and Conduit Schedule, Fluor Daniel Northwest, Richland, Washington.

H-1-83516, Rev. 2, FRS - KW Power Panels, Fluor Daniel Northwest, Richland, Washington.

H-1-83517, Rev. 2, FRS - KW Power Panels, Fluor Daniel Northwest, Richland, Washington.

H-1-83487, Rev. 1, FRS - KW Manipulator Support/Grating Modifications, Fluor Daniel Northwest, Richland, Washington.

H-1-83505, Rev. 2, Cable Tray and Conduit, Fluor Daniel Northwest, Richland, Washington.

H-1-83518, Rev. 1, FRS - KW EOC Ventilation, Fluor Daniel Northwest, Richland, Washington.

HNF-3525, Rev. 1, Design Package Lazy Susan for the FRS, Duke Engineering and Services Hanford, Richland, Washington. 


\section{SNF-5683, Revision 0}

HNF-4460, Rev. 1, Design Package Test Weights for Fuel Retrieval System, Duke Engineering and Services Hanford, Richland, Washington.

HNF-3579, Rev. 1, Design Package Vacuum Wand for Fuel Retrieval System, Duke Engineering and Services Hanford, Richland, Washington.

H-1-83990, Rev. 1, K Basin (FRS) Lazy Susan Fuel Basket Holder, Fluor Daniel Northwest, Richland, Washington.

H-1-83994, Rev. 1, FRS In-Pool Equipment Test Weight, Fluor Daniel Northwest, Richland, Washington.

H-1-83908, Rev. 0, FRS In-Pool Equipment Vacuum Wand Installation/Assy, Fluor Daniel Northwest, Richland, Washington.

H-1-83953, Rev. 0, Long Pole Tool Storage Rack/Holders, Fluor Daniel Northwest, Richland, Washington.

H-1-83907, Rev. 0, Broken Fuel Retrieval Tool - 2 modified Peter's Tools \#3, Fluor Daniel Northwest, Richland, Washington.

H-1-51829, Rev. 0, Sludge Depth Jacks, Fluor Daniel Northwest, Richland, Washington.

H-1-83905, Rev. 0, Fuel Stinger Installation/Removal Tool, Fluor Daniel Northwest, Richland, Washington.

H-1-84008, Rev. 0, Basket Annulus / Center Post Cover, Fluor Daniel Northwest, Richland, Washington.

H-1-83906, Rev. 0, Manipulator Extended Jaws, Fluor Daniel Northwest, Richland, Washington.

H-1-84013, Rev. 0, FRS Manipulator Hydraulic Splash Guard / Catch Pan, Fluor Daniel Northwest, Richland, Washington.

H-1-84056, Rev. 0, Broken Fuel Element Guide Pin, Fluor Daniel Northwest, Richland, Washington. 
SNF-5683, Revision 0

Appendix B

FRS Technical Document Status

B-1 


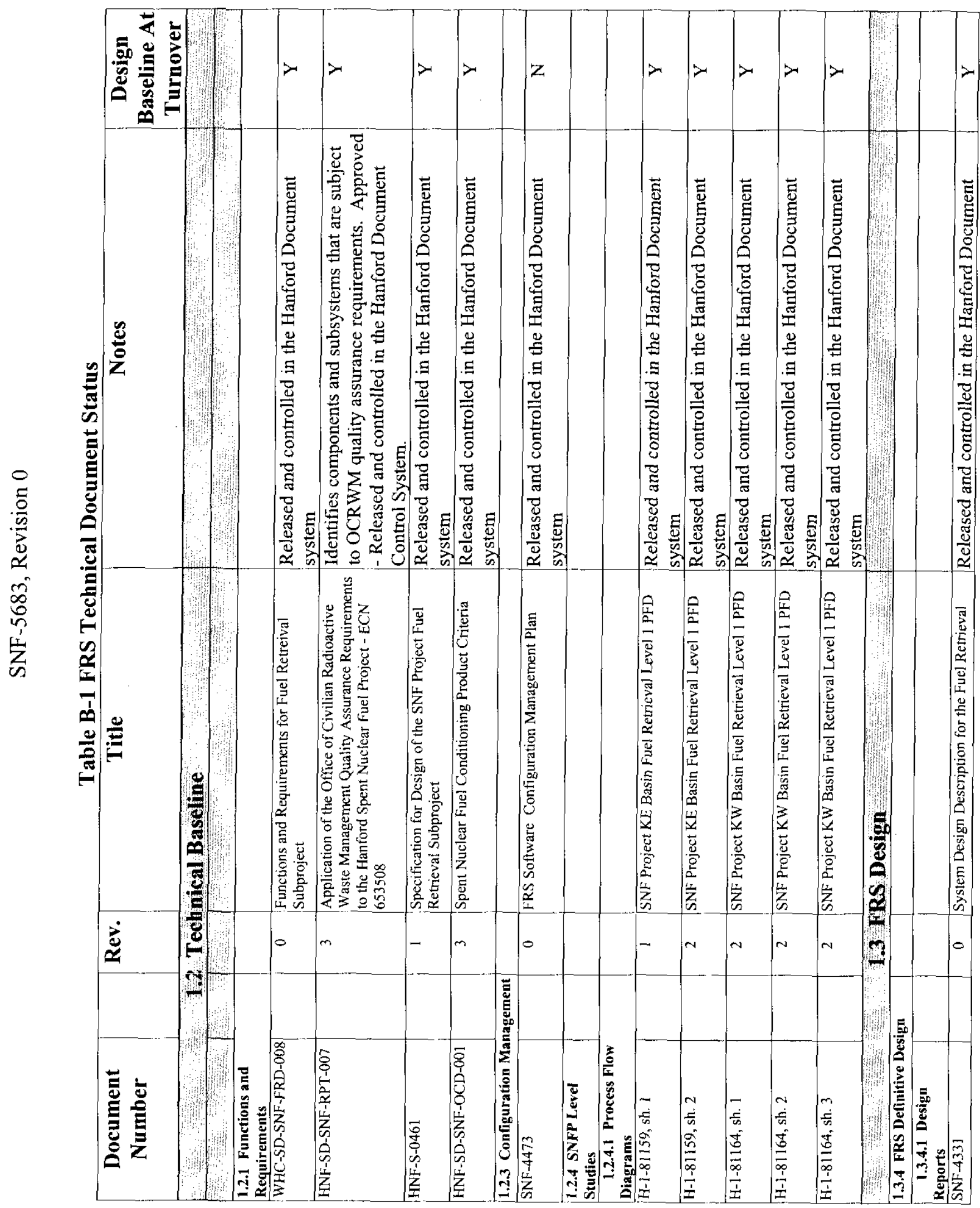

ஸे 


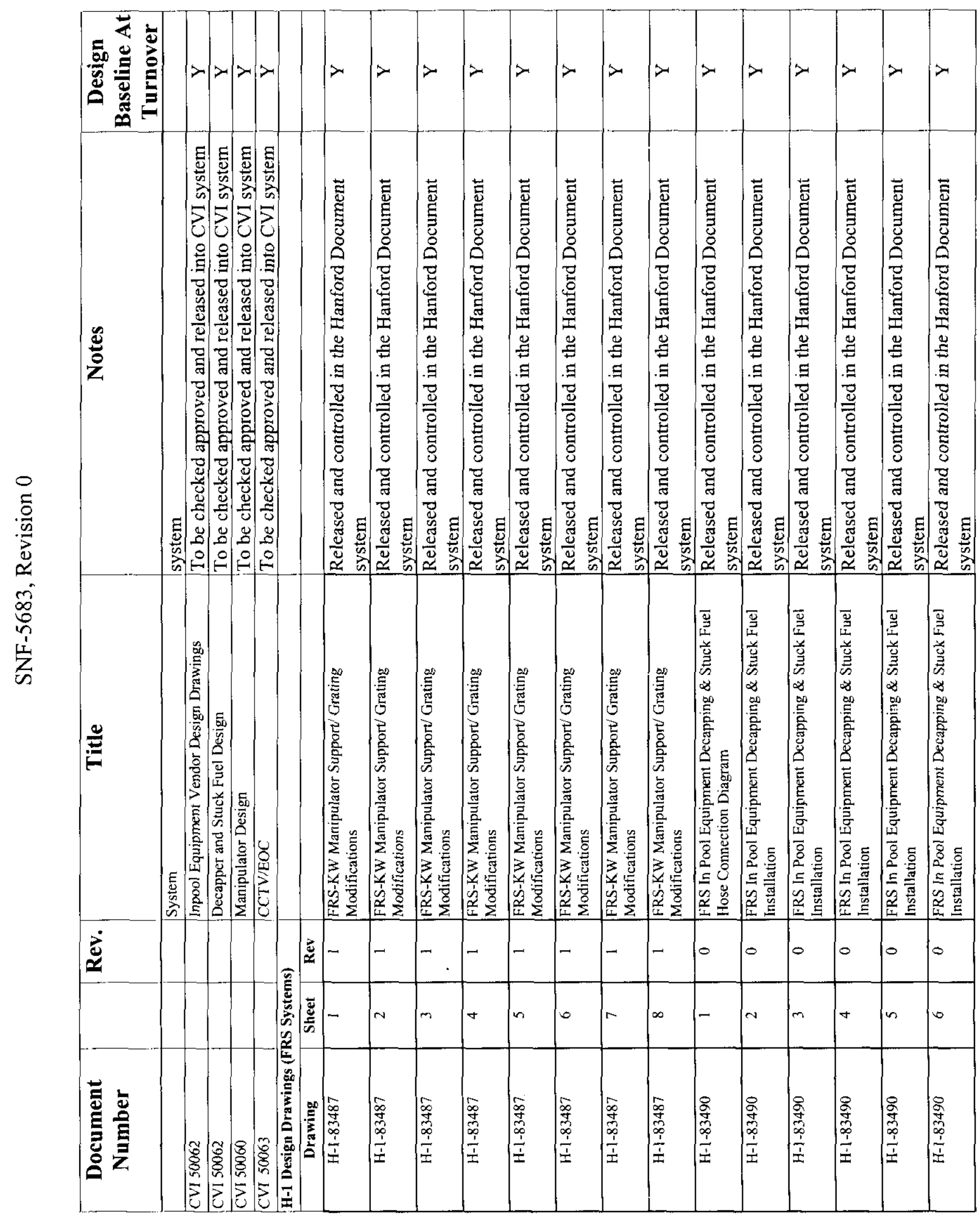

$\ddot{n}$ 


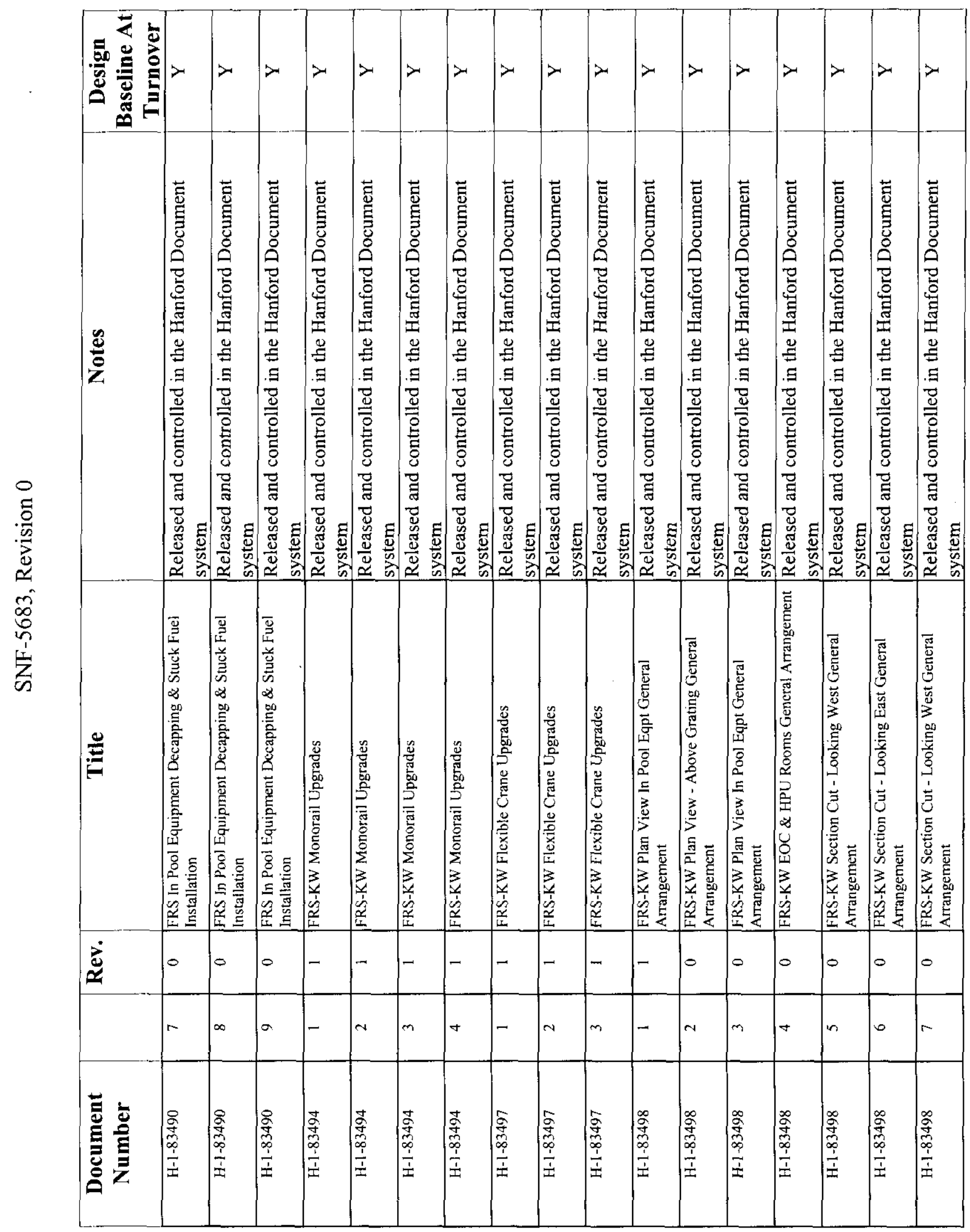

\$ 


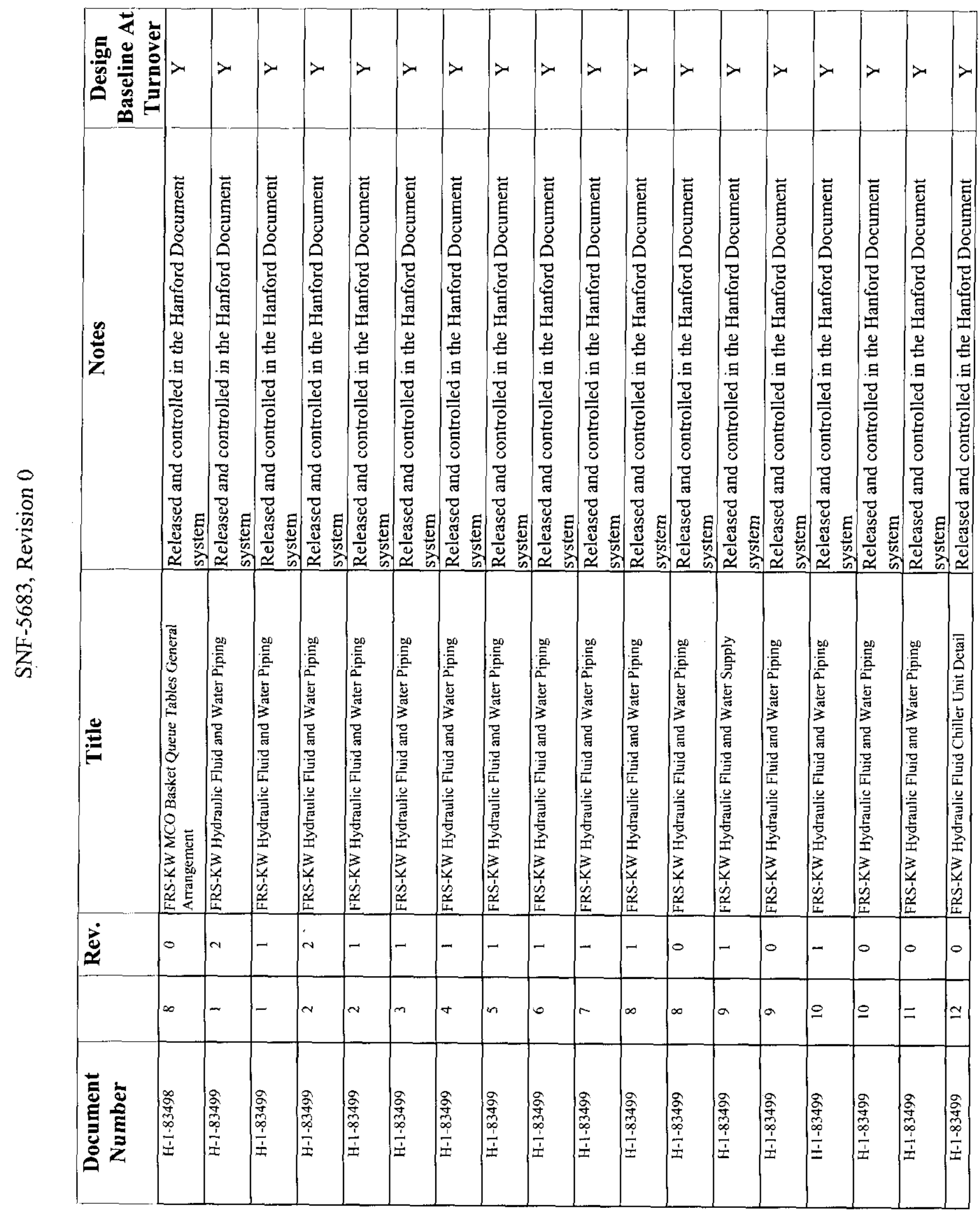




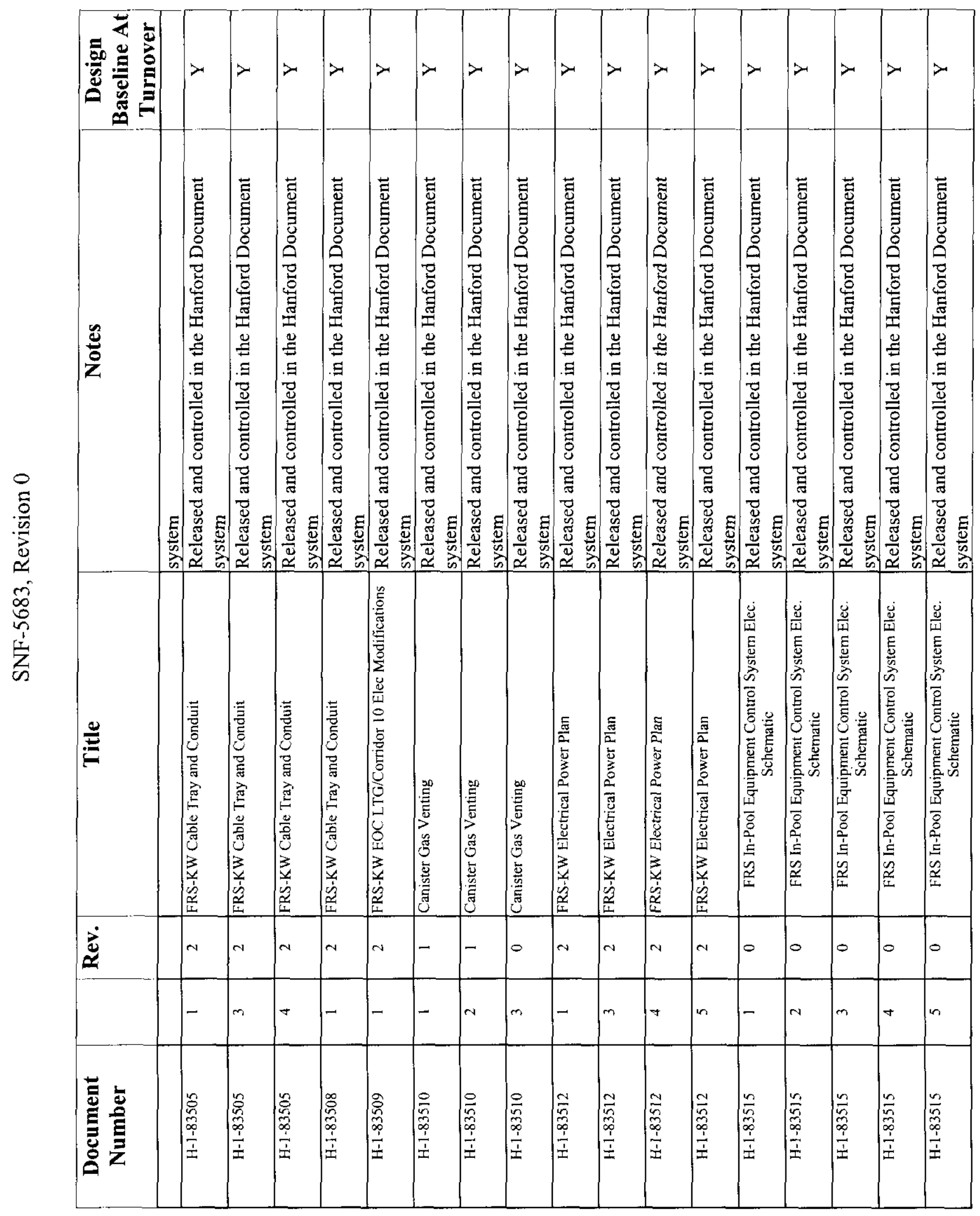

m 


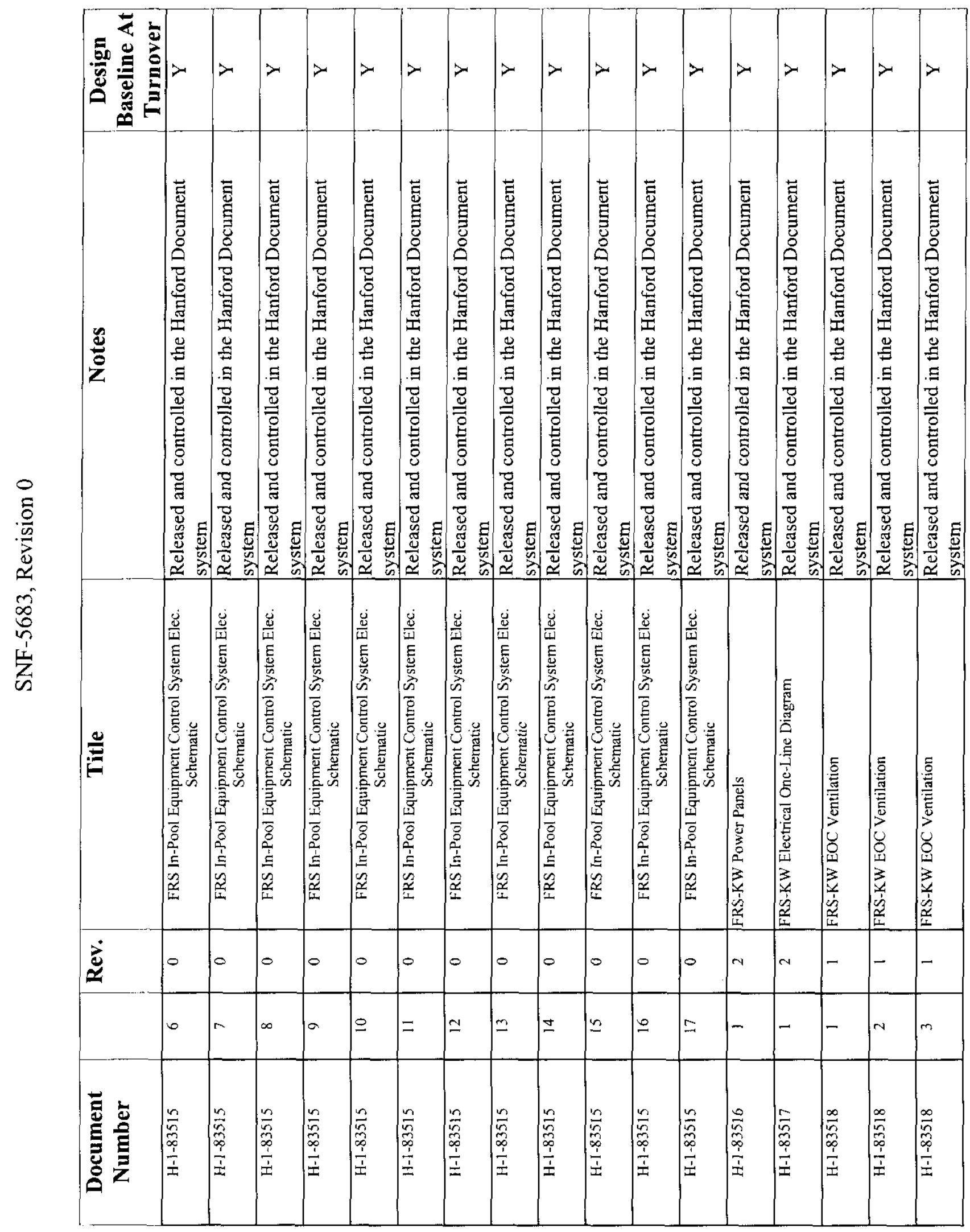

ले 


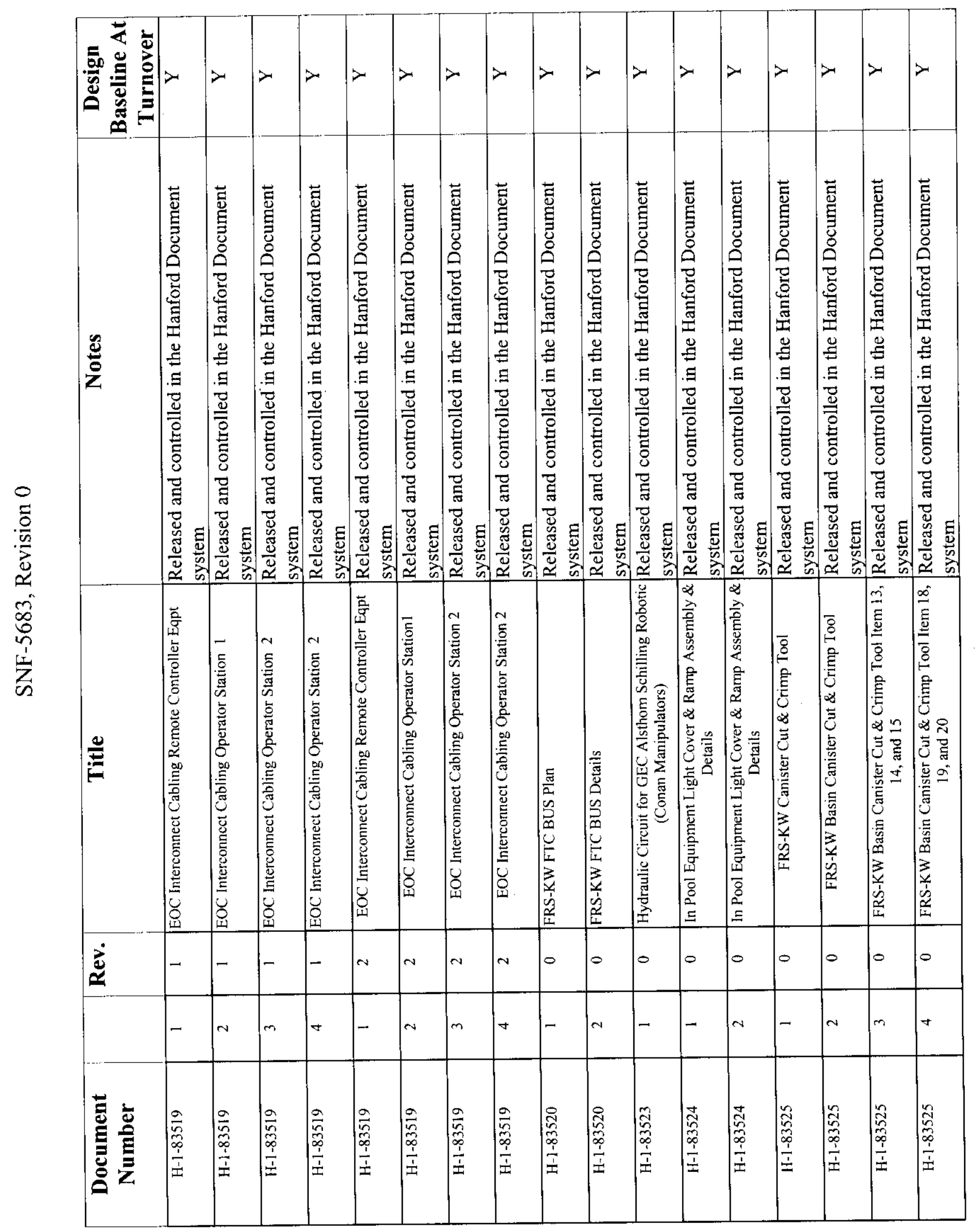




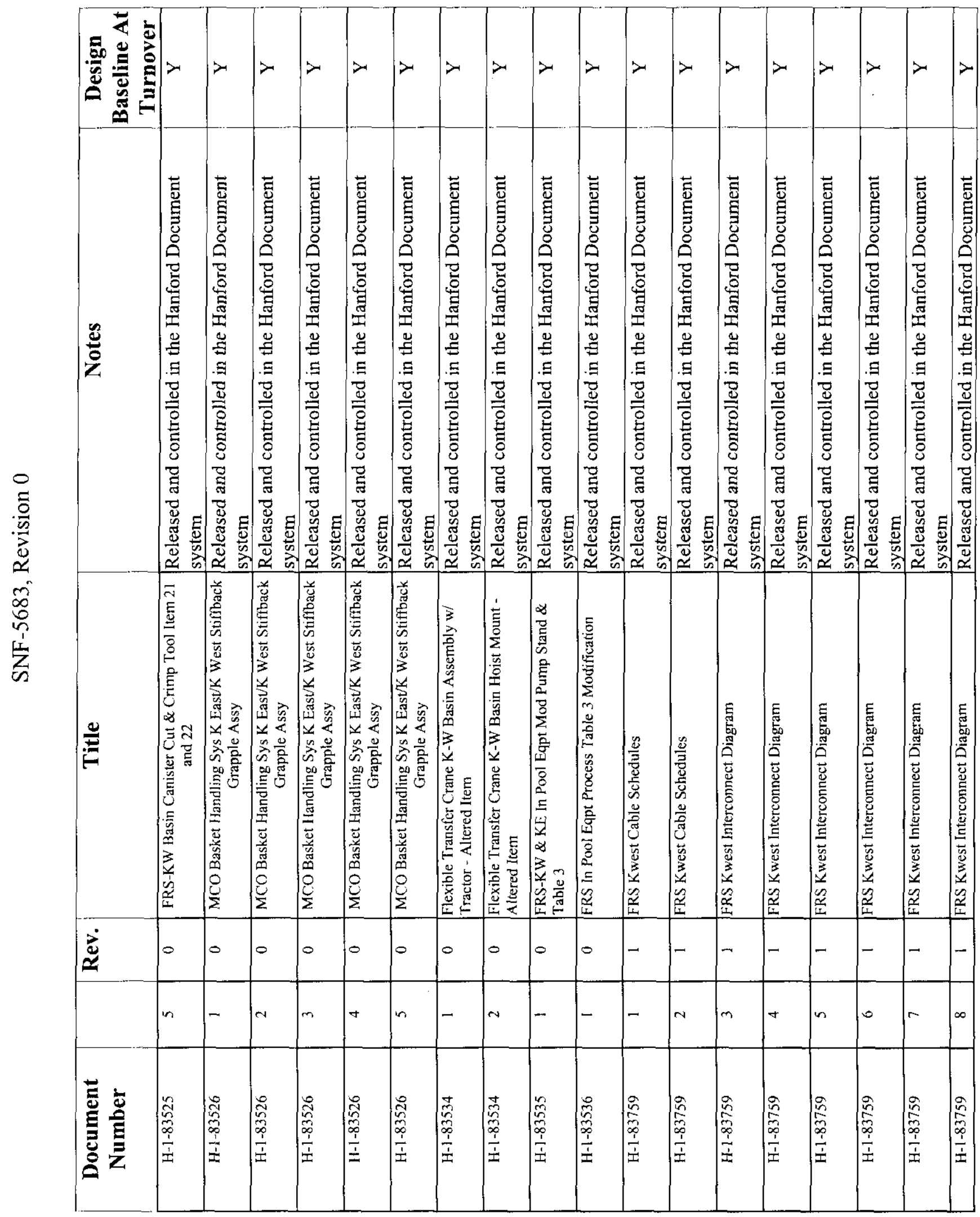




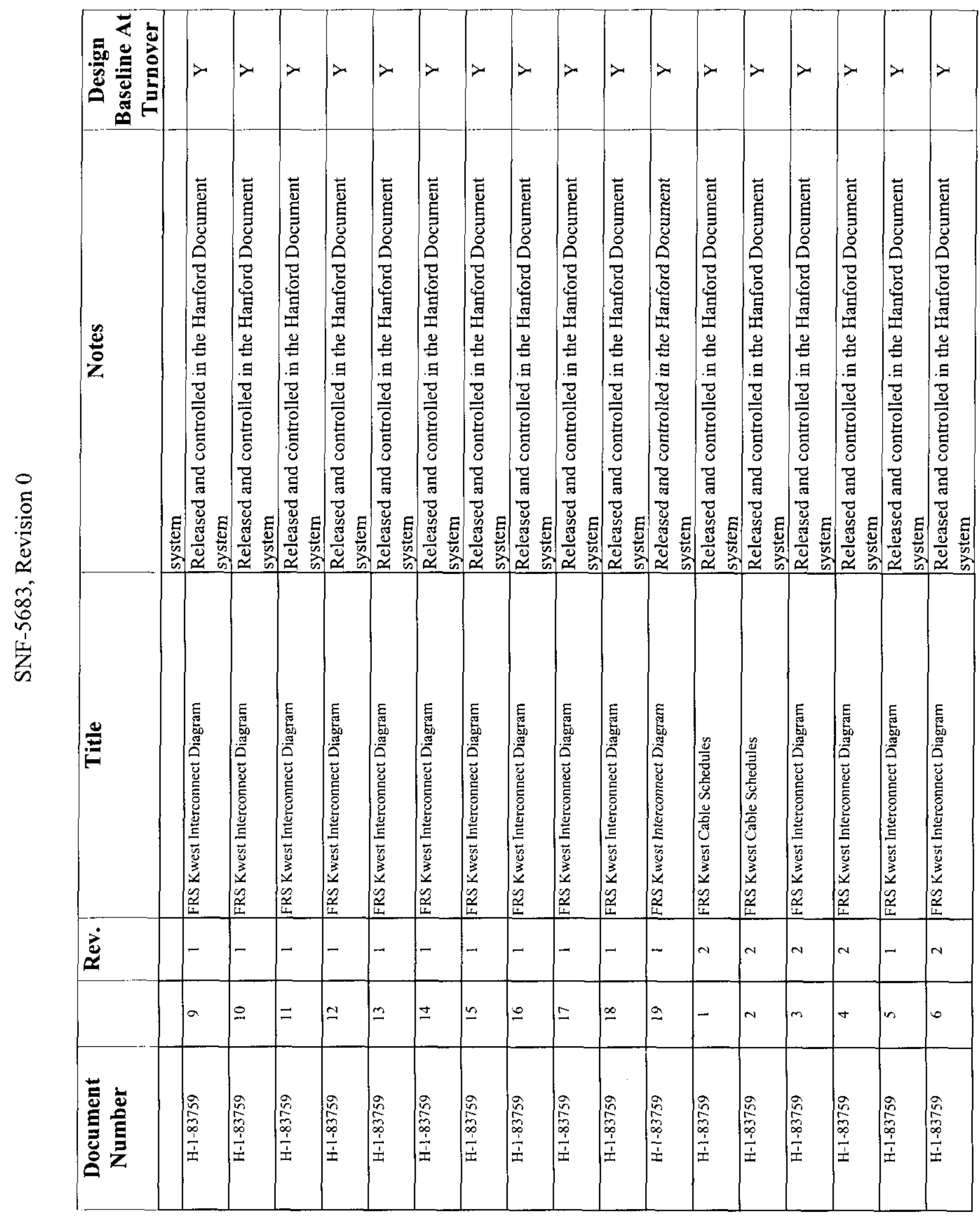




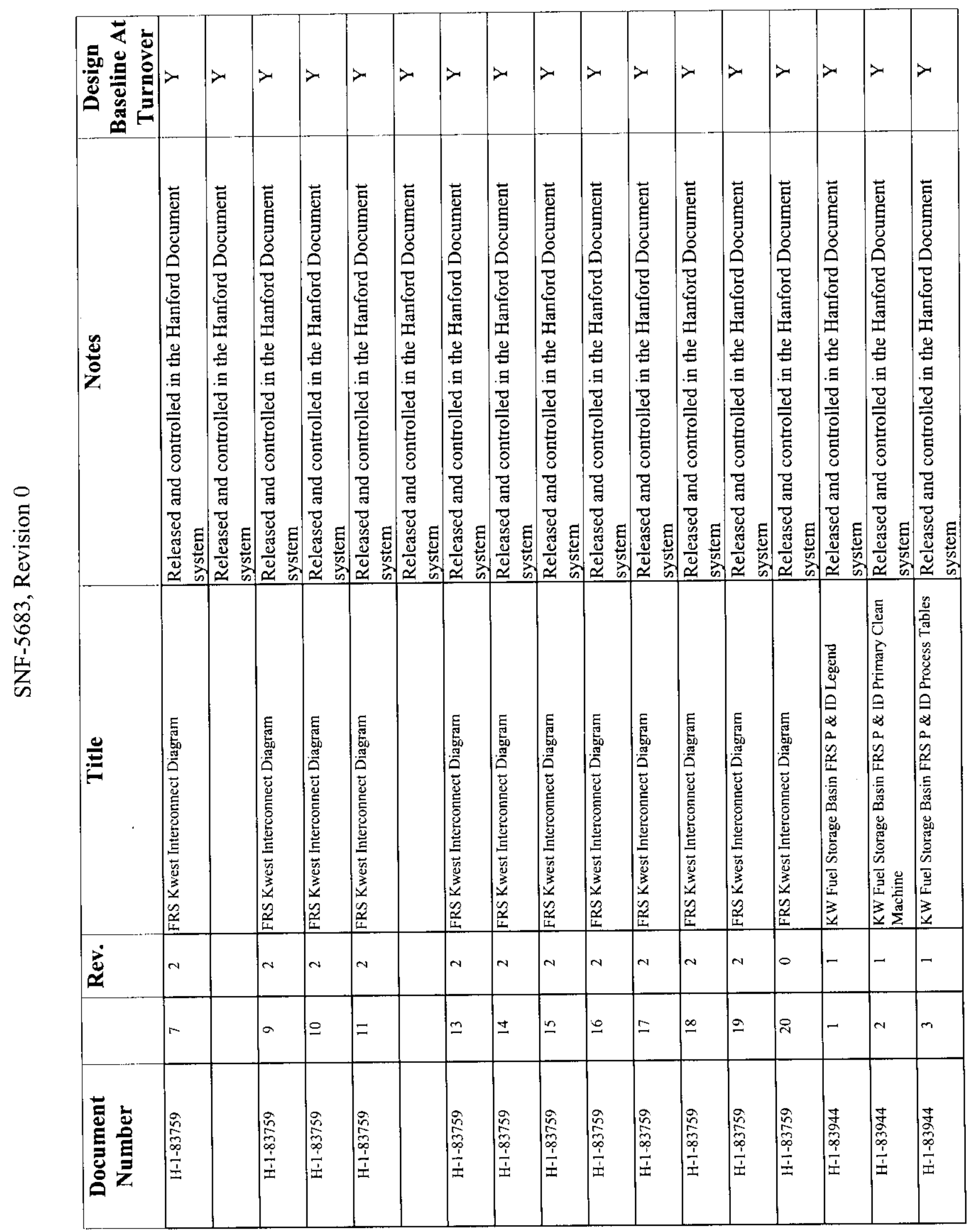

$\overline{\dot{x}}$ 


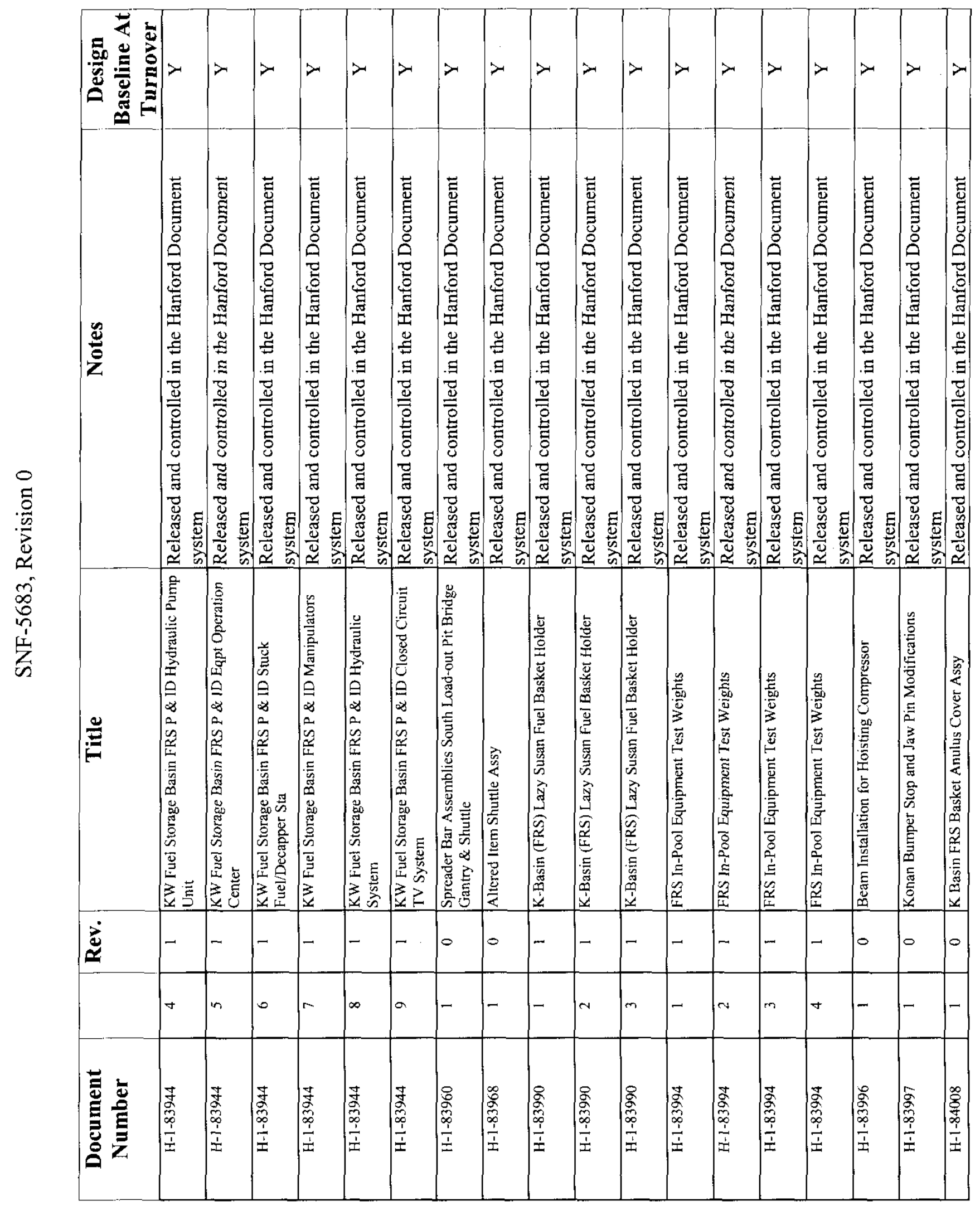




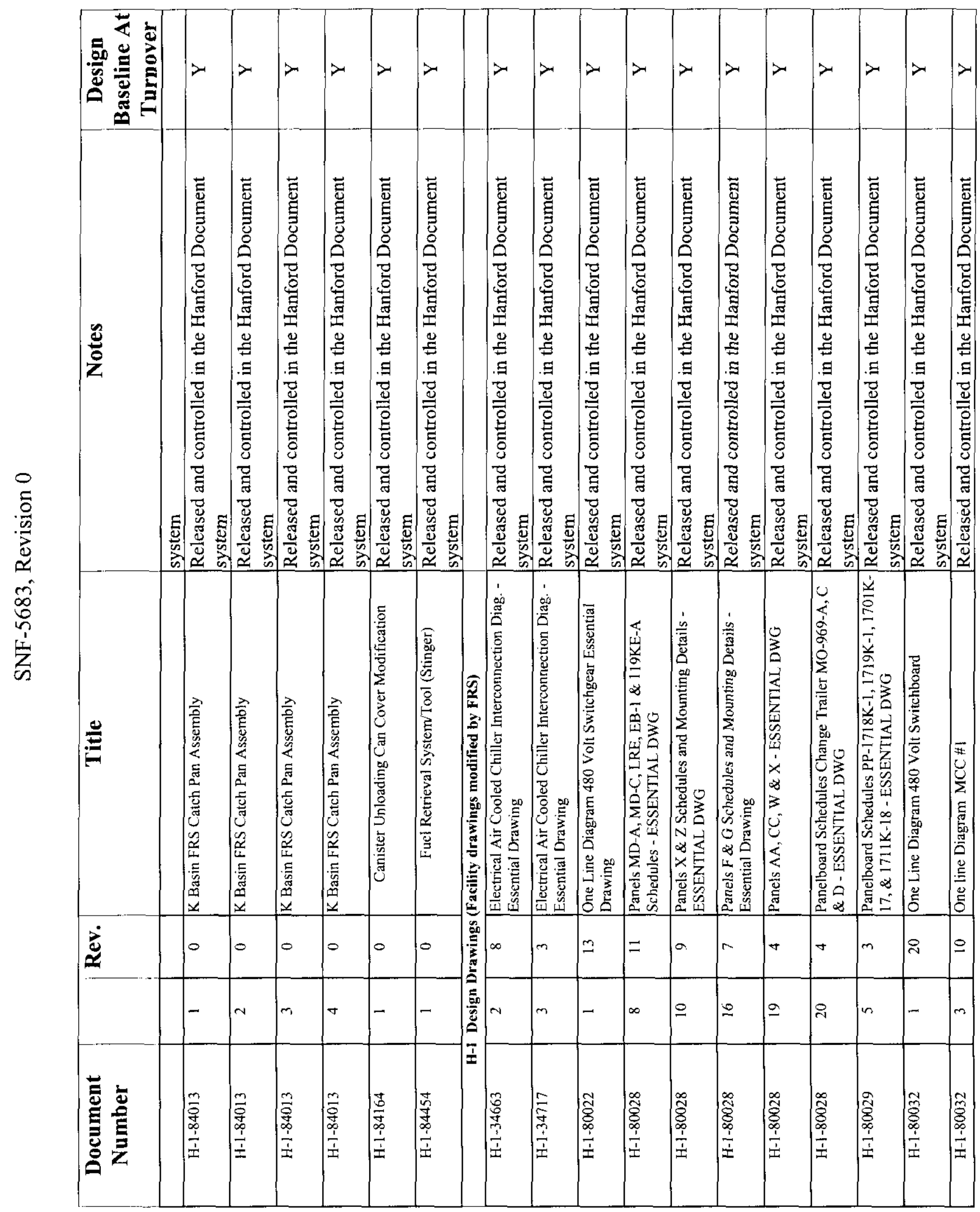




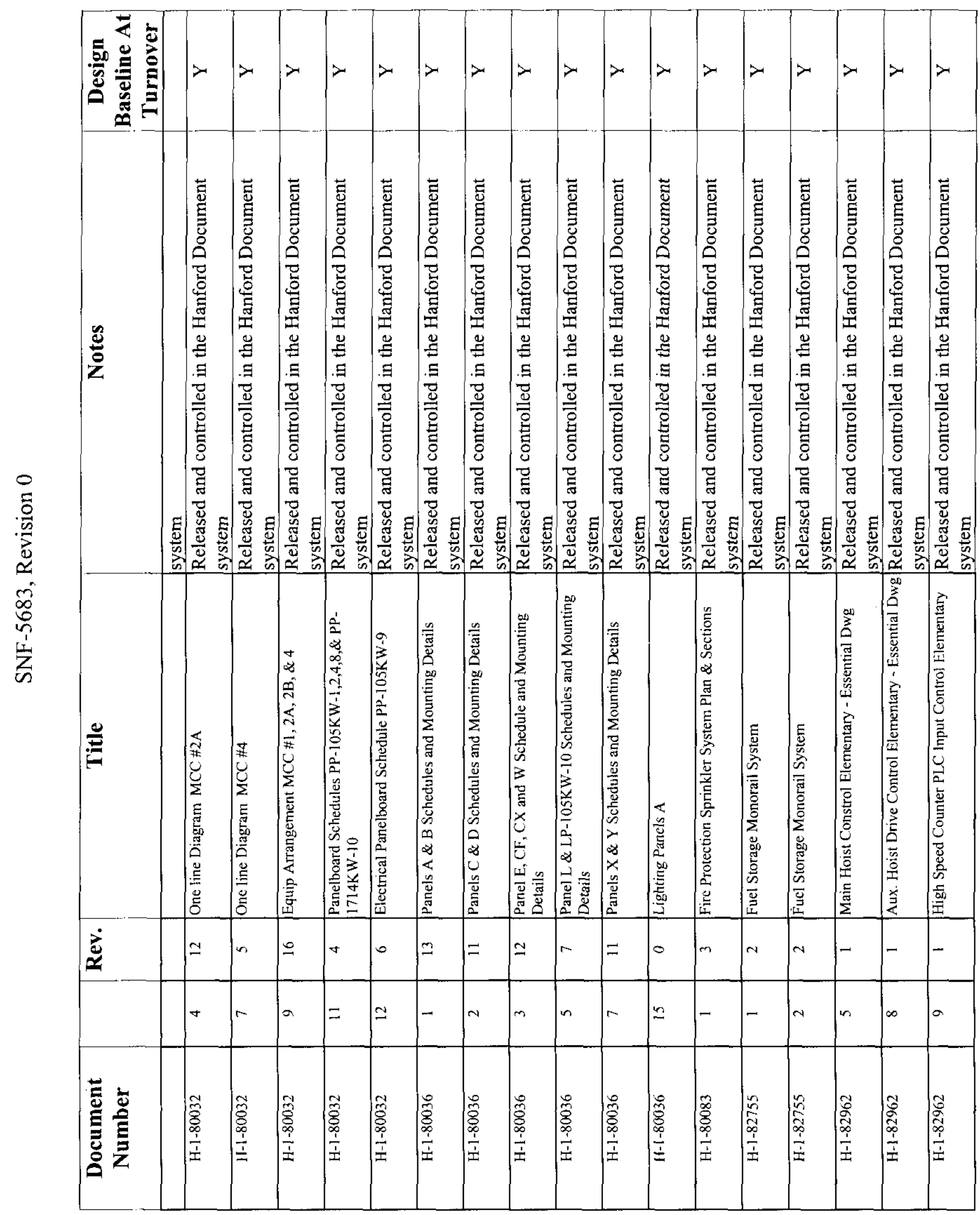




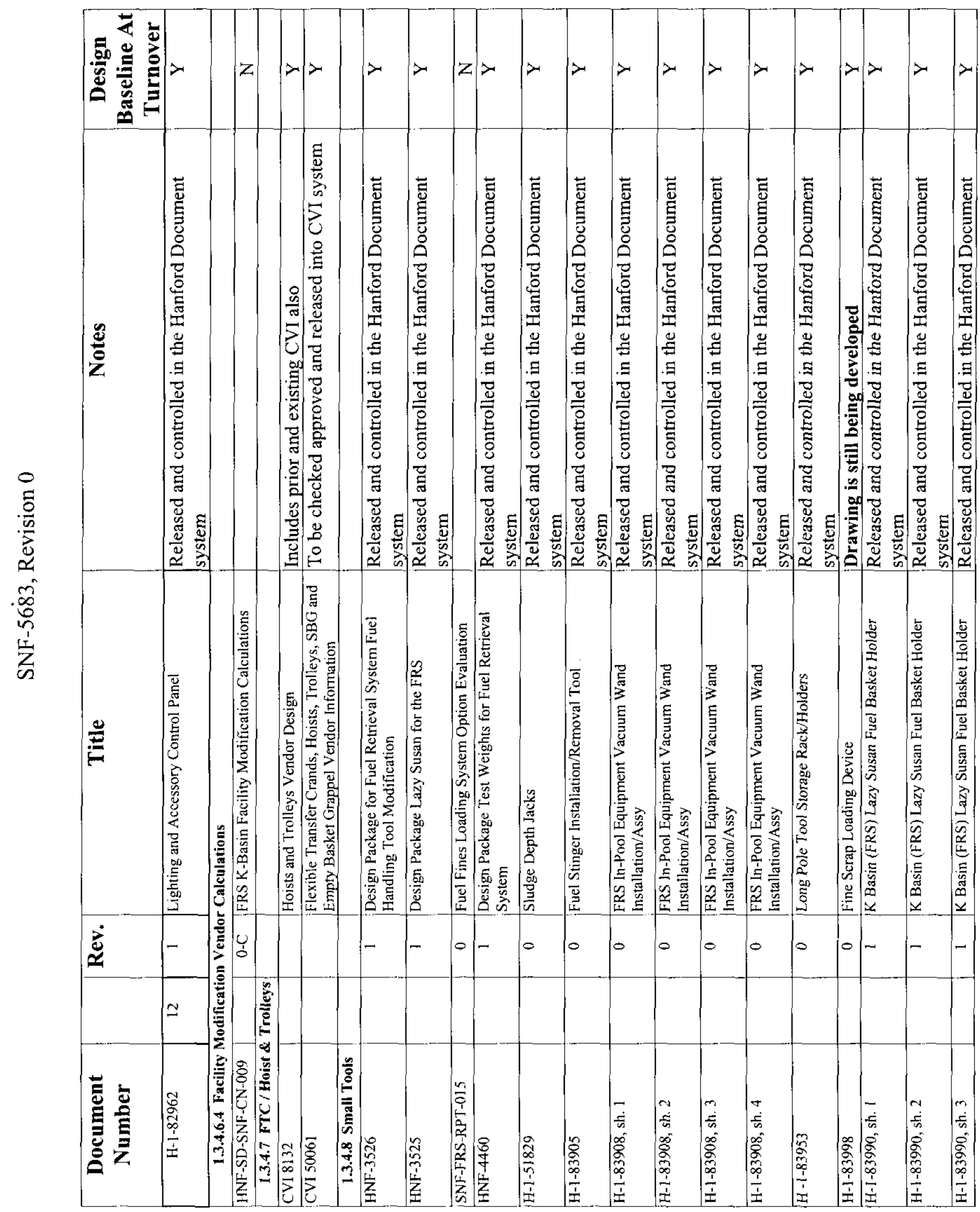




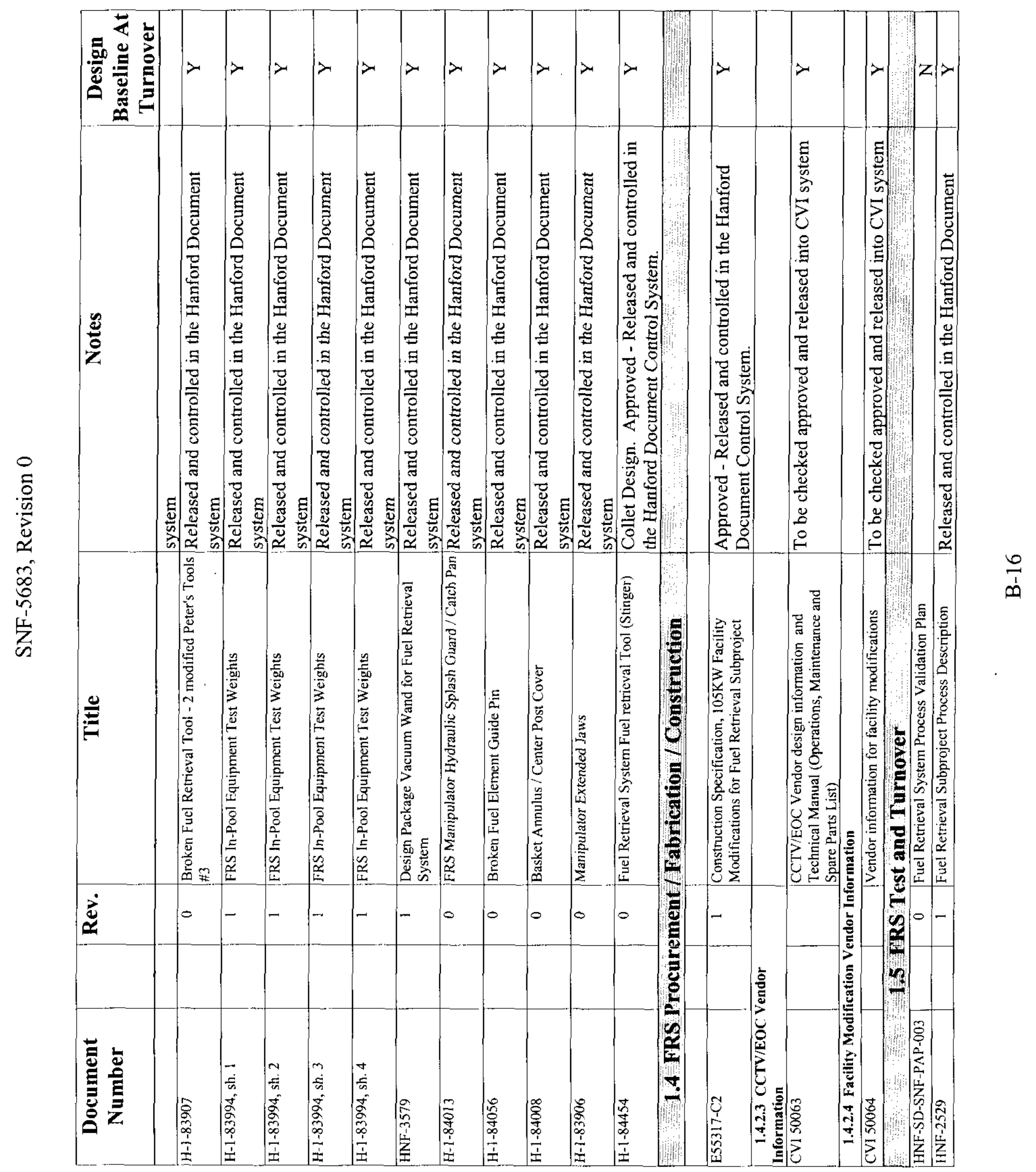




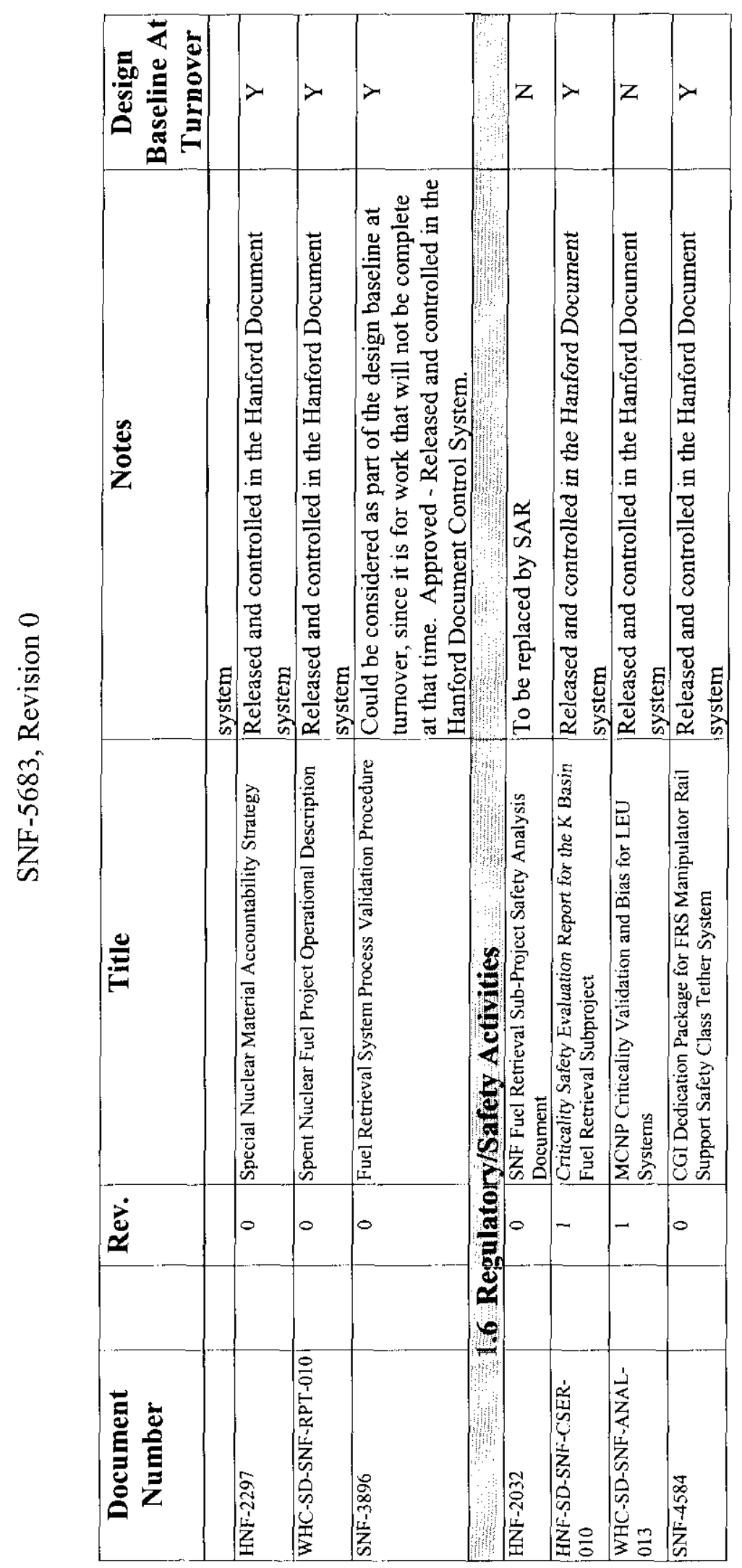




\section{Appendix C \\ Fuel Retrieval System ALARA and Radiological Design Basis and Review Summary}

SNF Project ALARA requirements are documented in the Spent Nuclear Fuel Project Technical Baseline Description, WHC-SD-SNF-SD-005, and associated Baseline Management System (BMS) database. FRS ALARA requirements are derived from the following:

a. 10 CFR 835, Occupational Radiation Protection,

b. DOE Order 6430.1A, General Design Criteria,

c. DOE/EH 0256T, U. S. Department of Energy Radiological Control Manual,

d. HSRCM-1, Hanford Site Radiological Manual,

e. WHC-SD-GN-30011, Radiological Design Guide, and

f. WHC-SD-SNF-SP-010, SNF Project Dose Management Plan.

These documents together with WHC-CM-4-29, Nuclear Criticality Safety, and WHC-CM-4-46, Non-reactor Facility Safety Analysis Manual, cover the FRS radiological safety design requirements which include ALARA requirements.

WHC-SD-SNF-PMP-016, Fuel Retrieval Subproject Project Management Plan (PMP), Section 7.3, assigns responsibility to the Design Authority (DA) for the establishment and configuration control of the FRS technical baseline is in accordance with the following safety assurance plans (Reference: PMP, Section 7.3.4):

a. Spent Nuclear Fuel Project Dose Management Plan, WHC-SD-SNF-SP-010, augmenting the plans set forth in the WHC Occupational ALARA Program, WHC-IP-1043.

b. Hanford Site Radiological Control Manual, HSRCM-1, providing the basis for FRS radiological safety assurance activities.

The FRS PMP, prepared in 1996, contained the top-level verification planning, including identification of requirements in need of verification, verification methods, verification responsibilities, stage of application, and verification product identification. FRS Radiological Design Review responsibility is assigned to the FRS Safety Engineer. This verification responsibility is documented in the FRS PMP, Section 6.3.7, Safety Engineer. Radiological Design Review is therefore documented by the Safety Engineer's signature on Engineering Data Transmittals (EDTs) and Engineering Change Notices (ECNs). The FRS Safety Engineer is responsible for:

1. "... assuring that the applicable safety disciplines have reviewed and concur with the safety elements of FRS products."

2. “... Review all FRS activities and products and assure that radiological safety policies, standards, and requirements are appropriately identified. Provide interpretation, where required, to assure that compliance will be achieved." 
Two verification methods were employed to assure SNF Project ALARA requirements were implemented in the FRS Subproject.

First, an informal review was performed on the FRS Subproject requirement baseline documented in WHC-SD-SNF-FRD-008, Functions and Requirements for Fuel Retrieval Subproject. (Verification Reference: EDT \#616105)

Second, an independent requirement traceability method of verification was used, as required by the FRS verification plan, (Reference: PMP, Section 7.3.1.) to further ensure implementation of SNF Project ALARA requirements, allocated to the FRS. That is, in WHC-SD-SNF-FRD-008, Functions and Requirements for Fuel Retrieval Subproject. The BMS captures ALARA requirement identification and implementation references. (Verification Reference: BMS Report dated February 26, 1998.)

The requirement traceability method of verification was also used, as required by the FRS verification plan, (Reference: PMP, Section 7.3.1.) to ensure implementation of ALARA requirements in lower tier FRS Project requirement documentation. Upon completion of the FRS requirement identification and analysis task, an independent Technical Requirements Review (TRR) was performed as required by the FRS verification plan. (Reference: PMP, Section 6.3.4.) The scope of this review included assurance that technical requirements, including ALARA requirements, from the FRS requirement identification and analysis task were captured in FRS Project sub-tier requirement documentation. That is, in HNF-S-0461, Specification for Design of the SNF Project Fuel Retrieval Subproject. This was part of the FRS "top-down" requirement traceability verification effort as described in SNF-5471, Appendix C, Section 2.0. The BMS captures ALARA requirement identification and HNF-S-0461 implementation references. (Verification Reference: BMS Report, dated February 26, 1998.)

FRS conceptual design was based upon design inputs which included radiological and ALARA requirements. These design inputs are documented in WHC-S-0438, Specification for Conceptual Design of the Spent Nuclear Fuel Retrieval Sub-Project System(s). This specification, verified via informal review, included a radiological design review as evidenced by the safety engineer's signature. (Verification Reference: EDT \#612987.) The Conceptual Design Report (CDR), L/B-SD-SNF-RPT-09, documents radiological and ALARA design inputs in Section 8.5, ALARA/Shielding. The CDR was verified via formal design review. (Verification References: 1) E. J. Shen 1996, Internal Memo to B. S. Carlisle, DESH, Approval of the 100\% Fuel Retrieval System Conceptual Design Report, 2C000-96-030, dated May 6, 1996; 2) E. J. Shen 1996, Internal Memo to B. S. Carlisle, DESH, Independent Design Review Report - Fuel Retrieval System 90\% Conceptual Design Report, 2C000-96-029, dated April 30, 1996.)

During the preliminary design stage, design products were verified via informal review. This included a radiological design review as evidenced by the safety engineer's signature. (Verification Reference Example: SNF-FRS-SPC-004, Performance Specification for Flexible Transfer Cranes and Process Hoist Systems, Revision 0, EDT \#606442.) Changes to approved design documentation was verified via informal review consistent with the original release. 
(Verification Reference Example: (SNF-FRS-SPC-004, Performance Specification for Flexible Transfer Cranes and Process Hoist Systems, Revision 1, ECN \#635037.)

A Radiological ALARA Design Implementation Plan, SNF-FRS-RPT-001, was completed which establishes a working and reference document standardizing the radiological ALARA design criteria, bases, methods, and activities. ALARA design verification was performed by Los Alamos Technical Associates (LATA). ALARA design verification is described in Section 4.2, Design Review. A reference document, standardized and formed the basis for radiological dose and shielding design activities is contained in L/B-SD-SNF-RPT-04, Radiological Shielding Design Plan for the SNF Fuel Retrieval Project. This document also establishes design verification responsibilities.

The FRS design agent, BNFL, performed an ALARA assessment of the FRS design. This assessment concluded that further radiological protection design features were not warranted. In addition, additional automation/remote operation of the FRS system has been shown not to be cost effective. The results are documented in SNF-FRS-RPT-012, Fuel Retrieval System ALARA Assessment. This assessment was verified via informal review including a radiological design review as evidenced by the safety engineer's signature. Verification documentation includes ALARA Chairperson. (Verification Reference: SNF-FRS-RPT-012, Fuel Retrieval System ALARA Assessment, EDT \#621105.)

The FRS design agent, British Nuclear Fuels Limited, Inc. (BNFL), scope of work included requirement traceability method of verification. The verification results were documented in SNF-FRS-RPT-009, Fuel Retrieval Sub-Project Compliance Evaluation Report (CER). This report documents radiological and ALARA requirement implementation references against the fuel retrieval radiological and ALARA requirements contained in specification HNF-S-0461. FRS project staff independently verified, and subsequently completed the CER, assuring that radiological and ALARA requirements were implemented in lower level technical documentation, such as design and/or procurement specifications, plans, assessments, etc. (Verification Reference: Internal Memo, R. M. Yanochko, SE, to E. J. Shen, FRS, Fuel Retrieval Subproject, Functions \& Requirements Traceability, 98-RMY-001, dated February 26, 1998.)

A final reconciliation of radiological and ALARA requirement traceability information contained in the CER was performed. This occurred when final procurement, fabrication specifications, etc., were completed. The CER was updated to reflect the current revision of performance specification HNF-S-0461, and final procurement specifications, etc. Radiological and ALARA requirement implementation references were subsequently verified and updated accordingly. (Verification Reference: Internal Memo, R. M. Yanochko, SE, to P. A. Young, FRS, Fuel Retrieval System, Functions \& Requirements Traceability - Compliance Evaluation Report, 99SNF/RMY-001, dated December 16, 1999.)

Changes to design documents were verified. Evidence of radiological design verification are documented by signatures on ECNs. (e.g., SNF-FRS-SPC-09, Revision 1, ECN \#635043) 


\section{DISTRIBUTION SHEET}

To

Distribution

Project Title/Work Order

Euel Retrieval Subproject/A-07

Name

T. B. Bergman

J. R. Cassidy

K. A. Colosi

G. M. Davis

J. J. Diehl

J. C. Fordham

G. D. Forehand

S. D. Godfrey (PEC) - 3 copies

B. D. Groth

J. M. Henderson

J. J. Klos

B. M. Koons

B. J. Knutson - Parsons

M. J. Langevin

D. R. Lucas

D. W. Medford

W. C. Miller

E. J. Muller

R. G. Pedigo

R. W. Rasmussen

R. J. Rourk

R. P. Ruth

L. S. Semmens

J. L. Shelor

R. T. Shupe

D. W. Siddoway

G. E. Stegen

J. A. Swenson

J. D. Thomson

J. L. Weamer

J. H. Wicks

M. J. Wiemers
From

Systems Engineering

Page 1 of 2
Date

EDT No. 623763

ECN No. N/A

\begin{tabular}{c|c|c|c|c|} 
MSIN & $\begin{array}{c}\text { Text } \\
\text { With All } \\
\text { Attach. }\end{array}$ & Text Only & $\begin{array}{c}\text { Attach./ } \\
\text { Appendix } \\
\text { Only }\end{array}$ & $\begin{array}{c}\text { EDT/ECN } \\
\text { Only }\end{array}$ \\
\hline
\end{tabular}




\section{DISTRIBUTION SHEET}

To

Distribution

Project Title $/$ Work Order

Fuel Retrieval Subproject/A-07

Name

R. M. Yanochko

P. A. Young

SNF Project File

FRS Project File

SNF Project Start-Up Library

SNF Project Training

SNF Project Procedures
From

Systems Engineering \begin{tabular}{ll} 
Page 2 of 2 \\
\hline Date
\end{tabular}

EDT No. 623763

ECN No. N/A

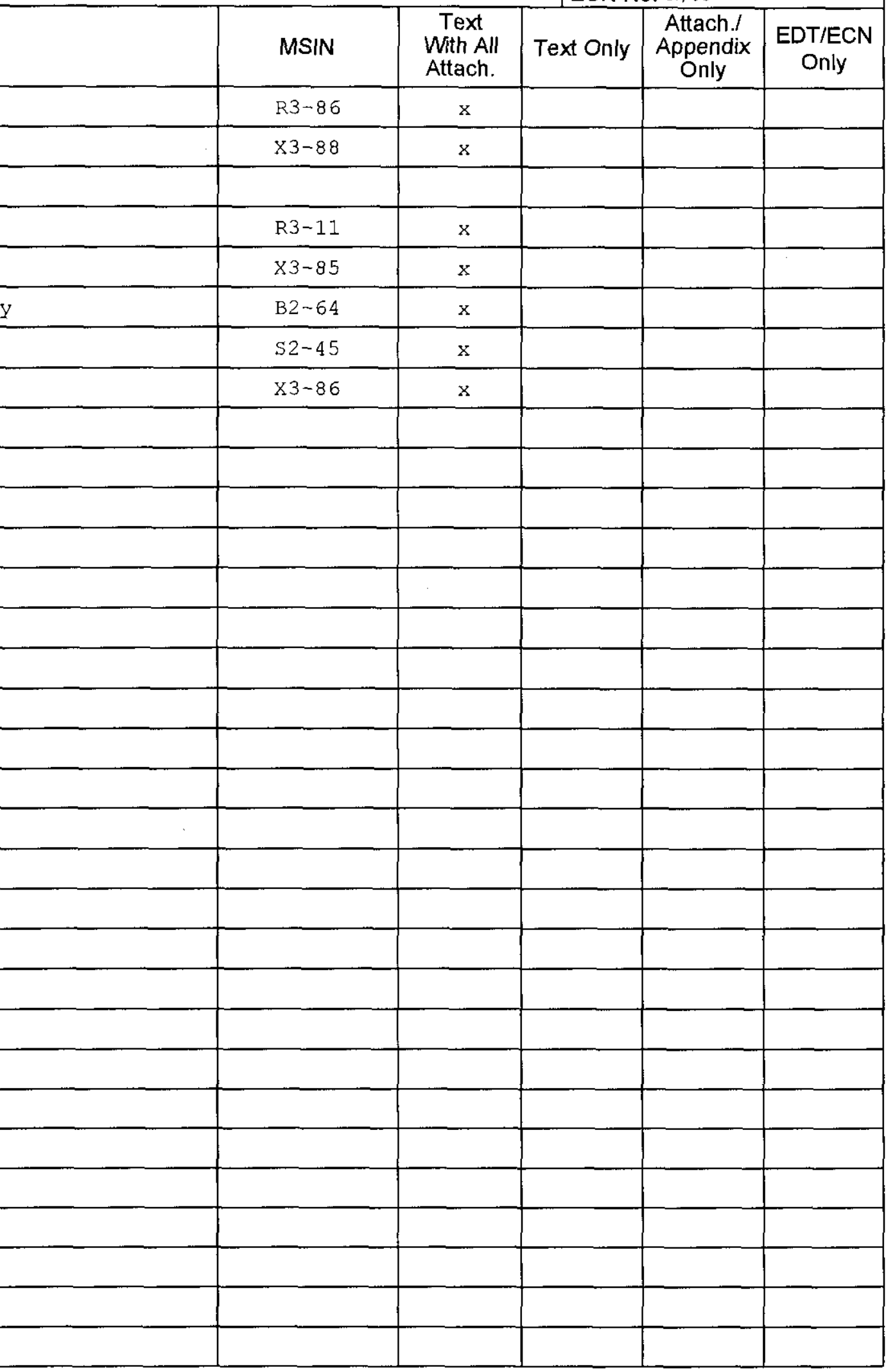

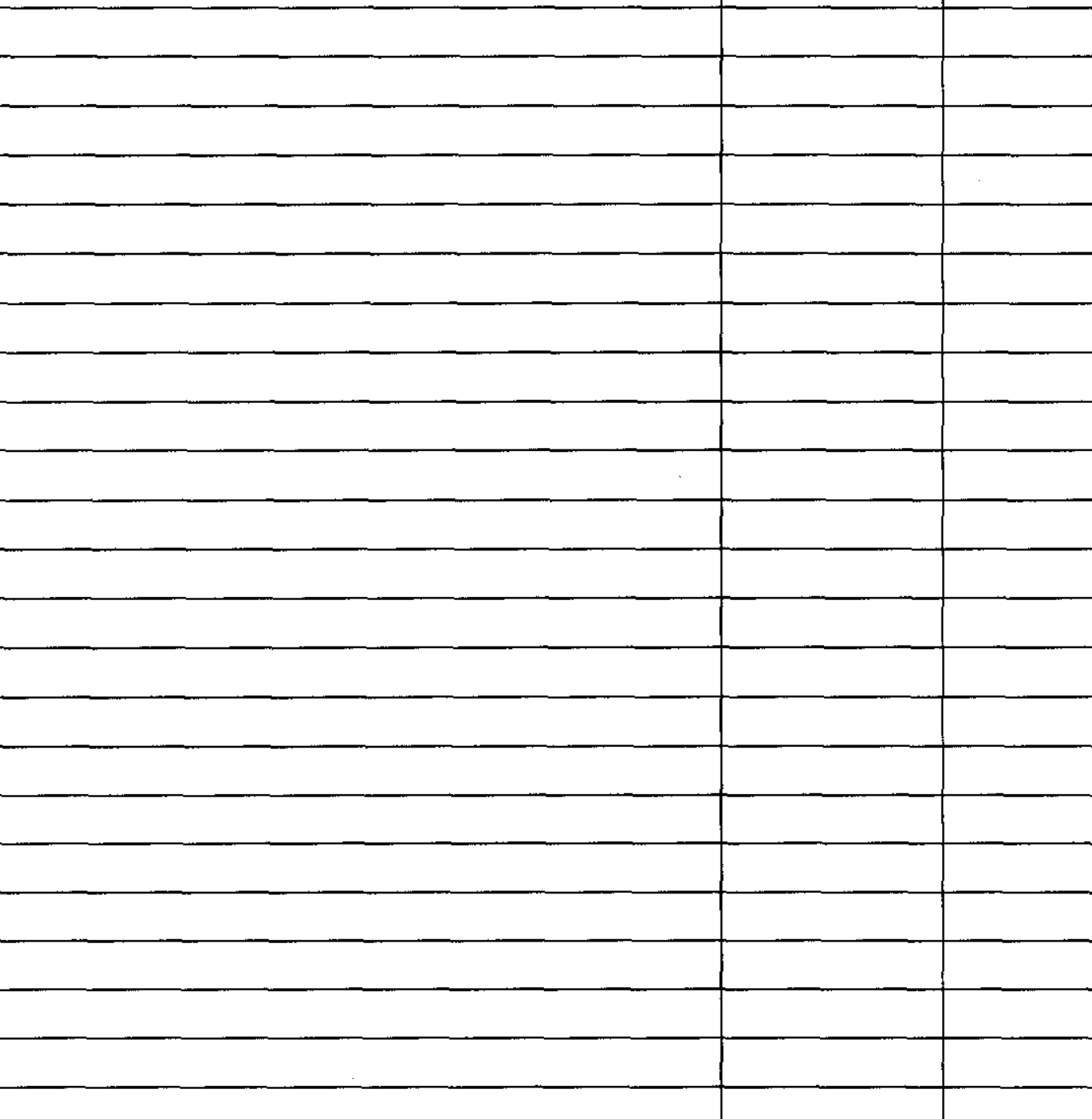

